\title{
Early evolution of the extraordinary Nova Delphini 2013 (V339 Del)^
}

\author{
A. Skopal ${ }^{1, \star \star}$, H. Drechsel ${ }^{2}$, T. Tarasova ${ }^{3}$, T. Kato ${ }^{4}$, M. Fujii ${ }^{5}$, F. Teyssier ${ }^{6}$, O. Garde $^{7}$, J. Guarro $^{8}$, J. Edlin ${ }^{9}$, C. Buil ${ }^{10}$,
} D. Antao ${ }^{11}$, J.-N. Terry ${ }^{12}$, T. Lemoult ${ }^{13}$, S. Charbonnel ${ }^{14}$, T. Bohlsen ${ }^{15}$, A. Favaro ${ }^{16}$, and K. Graham ${ }^{17}$

1 Astronomical Institute, Slovak Academy of Sciences, 05960 Tatranská Lomnica, Slovakia e-mail: skopal@astro.sk

2 Dr. Karl Remeis-Observatory \& ECAP, Astronomical Institute, Friedrich-Alexander-Universität Erlangen-Nürnberg, Sternwartstraße 7, 96049 Bamberg, Germany

3 Crimean Astrophysical Observatory, 298409 Nauchny, Crimea, Russia

4 Department of Astronomy, Kyoto University, Kitashirakawa-Oiwake-cho, Sakyo-ku, 606-8502 Kyoto, Japan

5 Fujii Kurosaki Observatory, 4500 Kurosaki, Tamashima, Kurashiki, 713-8126 Okayama, Japan

${ }^{6} 67$ rue Jacques Daviel, 76100 Rouen, France

7 Observatoire de la Tourbière, 38690 Chabons, France

8 Balmes 2, 08784 Piera, Barcelona, Spain

91833 Bobwhite Dr. Ammon, Idaho, 83401, USA

10 Castanet Tolosan Observatory, 6 place Clemence Isaure, 31320 Castanet Tolosan, France

${ }^{11}$ Lieu-dit Durfort, 81150 Fayssac, France

126 rue Virgile, 42100 Saint-Étienne, France

13 Chelles Observatory, 23 avenue Hénin, 77500 Chelles, France

14 Durtal Observatory, 6 rue des Glycines, 49430 Durtal, France

15 Mirranook Observatory, Boorolong Rd Armidale, NSW, 2350, Australia

16 Société Astronomique de Bourgogne-Dijon, France

1723746 Schoolhouse Road, Manhattan, Illinois, 60442, USA

Received 27 May 2014 / Accepted 25 July 2014

\section{ABSTRACT}

\begin{abstract}
Aims. We determine the temporal evolution of the luminosity $\left(L_{\mathrm{WD}}\right)$, radius $\left(R_{\mathrm{WD}}\right)$ and effective temperature $\left(T_{\text {eff }}\right)$ of the white dwarf (WD) pseudophotosphere of V339 Del from its discovery to around day 40. Another main objective was studying the ionization structure of the ejecta.

Methods. These aims were achieved by modelling the optical/near-IR spectral energy distribution (SED) using low-resolution spectroscopy (3500-9200 $\AA$ ), $U B V R_{\mathrm{C}} I_{\mathrm{C}}$ and $J H K L M$ photometry. Important insights in the physical conditions of the ejecta were gained from an analysis of the evolution of the $\mathrm{H} \alpha$ and Raman-scattered $6825 \AA$ O VI line using medium-resolution spectroscopy $(R \sim 10000)$.

Results. During the fireball stage (Aug. 14.8-19.9, 2013), $T_{\text {eff }}$ was in the range of 6000-12 $000 \mathrm{~K}$, $R_{\mathrm{WD}}$ was expanding non-uniformly in time from $\sim 66$ to $\sim 300(d / 3 \mathrm{kpc}) R_{\odot}$, and $L_{\mathrm{WD}}$ was super-Eddington, but not constant. Its maximum of $\sim 9 \times 10^{38}(d / 3 \mathrm{kpc})^{2} \mathrm{erg} \mathrm{s}^{-1}$ occurred around Aug. 16.0, at the maximum of $T_{\text {eff }}$, half a day before the visual maximum. After the fireball stage, a large emission measure of $1.0-2.0 \times 10^{62}(\mathrm{~d} / 3 \mathrm{kpc})^{2} \mathrm{~cm}^{-3}$ constrained the lower limit of $L_{\mathrm{WD}}$ to be well above the super-Eddington value. The mass of the ionized region was a few $\times 10^{-4} M_{\odot}$, and the mass-loss rate was decreasing from $\sim 5.7$ (Aug. 22) to $\sim 0.71 \times 10^{-4} M_{\odot} \mathrm{yr}^{-1}$ (Sept. 20). The evolution of the $\mathrm{H} \alpha$ line and mainly the transient emergence of the Raman-scattered O VI $1032 \AA$ A line suggested a biconical ionization structure of the ejecta with a disk-like H I region persisting around the WD until its total ionization, around day 40. On Sept. 20 (day 35), the model SED indicated a dust emission component in the spectrum. The dust was located beyond the H I zone, where it was shielded from the hard, $\gtrsim 10^{5} \mathrm{~K}$, radiation of the burning WD at that time.

Conclusions. Our extensive spectroscopic observations of the classical nova V339 Del allowed us to map its evolution from the very early phase after its explosion. It is evident that the nova was not evolving according to the current theoretical prediction. The unusual non-spherically symmetric ejecta of nova V339 Del and its extreme physical conditions and evolution during and after the fireball stage represent interesting new challenges for the theoretical modelling of the nova phenomenon.
\end{abstract}

Key words. novae, cataclysmic variables - stars: fundamental parameters - stars: individual: V339 Del

\section{Introduction}

According to the first report on Nova Delphini 2013 (PNV J20233073+2046041, V339 Del), announced by the Central Bureau Electronic Telegram No. 3628, the nova was discovered by Koichi Itagaki on 2013 Aug. 14.584 UT at the unfiltered brightness of $6.8 \mathrm{mag}$, and its progenitor was identified

$\star$ Based on data collected by amateur astronomers.

$\star \star$ Visiting Astronomer: Astronomical Institute, Bamberg. by Denisenko and collaborators as the blue star USNO-B1.0 1107-0509795 ( $B \sim 17.2-17.4, R \sim 17.4-17.7)$. First optical spectra were obtained on 2013 Aug. 14.844 by observers participating in the Astronomical Ring for Access to Spectroscopy (ARAS) project $^{1}$ (Shore et al. 2013a) and by Darnley et al. (2013) on 2013 Aug. 14.909.

\footnotetext{
1 http://www.astrosurf.com/aras/Aras_DataBase/Novae/ Nova-Del-2013.htm
} 
A first description of the multicolour optical photometry was provided by Munari et al. (2013a). They estimated the peak brightness of V339 Del to $V \sim 4.43$, reached at Aug. 16.44 UT, that is, $\sim 1.85$ days after its discovery. An increase of the observed colour index $B-V$ from $\sim 0.1$ to $\sim 0.55$ between Aug. 15.0 and 19.2 (see Fig. 2 of Munari et al. 2013a) corresponded to a change of the spectral type of the expanding envelope from $\sim \mathrm{A} 2$ to $\sim \mathrm{F} 8$ (cf. Cox 2000). The following gradual decrease of the $B-V$ index after Aug. 20 suggested a dramatic change of the optical spectrum of the nova.

Spectroscopic observations during this early stage of the nova evolution were consistent with its photometric behaviour. Tarasova \& Shakhovskoi (2013) reported that the spectral energy distribution (SED) of their pre-maximum spectrum, taken on Aug. 15.8, was similar to a late-A or early-F-type spectrum. The line spectrum, which was dominated by P Cyg-type hydrogen lines with a pronounced absorption component (see Darnley et al. 2013; Shore et al. 2013a; Tomov et al. 2013; Tarasova 2013; Munari et al. 2013b), also suggested that the effective temperature of the envelope probably is $\lesssim 10^{4} \mathrm{~K}$. During Aug. 19, Darnley \& Bode (2013) observed a significant weakening of the H I absorption components, while those of the Fe II, He I, and O I profiles were still clearly present. This evolution indicated a distinctive cooling of the expanding envelope. In the continuum, the temperature decrease was manifested by a flattening of the optical SED almost without traces of the Balmer jump, as observed by Tarasova \& Shakhovskoi (2013) on Aug. 19.9. This behaviour signalized the end of the early optically thick phase (also called the fireball or iron-curtain stage, see Shore 2008), when the expanding shell transfers the inner energetic photons to its optically thick/thin interface. When the nova shell reaches a maximum radius, the optical depth of its outer parts starts to decrease, and the white dwarf (WD) pseudophotosphere progressively shrinks and becomes hotter. As a result, the spectrum significantly changes in both the continuum and lines, shifting the maximum of its SED to shorter wavelengths. For nova V339 Del this transition occurred during Aug. 20-22, as described by Shore et al. (2013b).

In this paper we describe the evolution of the fundamental parameters, $L_{\mathrm{WD}}, R_{\mathrm{WD}}$, and $T_{\text {eff }}$ of nova V339 Del from its detection to the onset of a stage with a harder spectrum (around day 40, when the first significant X-ray flux occurred). We note that the Raman-scattered $6825 \AA$ line was observed for the first time in the spectrum of a classical nova, while it is commonly identified only for symbiotic systems. The variation of the $\mathrm{H} \alpha$ and Raman $6825 \AA$ line profiles allowed us to determine the ionization structure of the ejecta during this early period of the nova evolution. Section 3 presents the results, which are interpreted and summarized in Sects. 4 and 5.

\section{Observations}

Spectroscopic observations of V339 Del were secured at different observatories and/or private stations:

(i) At the Crimean Astrophysical Observatory with the $2.6 \mathrm{~m}$ Shajn telescope, using a SPEM spectrograph in the Nasmith focus; the detector was a SPEC-10 CCD camera $(1340 \times 100$ pixel $)$.

(ii) At the Fujii Kurosaki Observatory with a $0.4 \mathrm{~m}$ SCT F10 (Meade) telescope, using a FBSPEC-III spectrograph and a CCD camera ML6303E(FLI) $(3072 \times 2048$ pixel $)$ as the detector. (iii) At the Observatory de la Tourbière with a $0.355 \mathrm{~m}$ Schmidt-Cassegrain telescope (Celestron C14) equipped with an eShel spectrograph (Shelyak; optical fiber of $50 \mu \mathrm{m}$ ) mounted at the $f / \mathrm{d} 7$ focus. The detector was an ATIK 460EX CCD camera (pixel size of $4.54 \mu \mathrm{m}$, binning $2 \times 2$ mode; pixel of $9.08 \mu \mathrm{m}$ ).

(iv) At the Santa Maria de Montmagastrell Observatory, Tàrrega (Lleida) Spain, using a Control Remote Telescope SC16 equipped with a spectrograph B60050-VI; the detector was an ATIK 460EX CCD camera.

(v) At a private station in Idaho Falls with a $0.35 \mathrm{~m}$ Celestron C14 telescope, using a LISA spectrograph from Shelyak Instruments; the detector was an ATIK 460E CCD camera.

(vi) At the Castanet Tolosan Observatory with a $0.2 \mathrm{~m} \mathrm{~F} 4$ Newton telescope, using the eShel model of the echelle spectrograph (Shelyak) and a CCD camera ATIK 640EX. On Oct. 1.923 2013, a 0.28 m F6 SCT telescope was used.

(vii) At the private station Fayssac with a $0.25 \mathrm{~m}$ telescope, using the Alpy 600 spectrograph (Shelyak) in the Newton focus; the detector was an ATIK 314L CCD camera.

(viii) At the Observatoire du Pilat, with a $0.25 \mathrm{~m} \mathrm{LX} 200$ (Meade) telescope, using the Alpy 600 spectrograph (Shelyak); the detector was SBIG ST-8300 CCD camera wit a Kodak KAF 8300 chip.

(ix) At the private station Chelles, with a $0.35 \mathrm{~m}$ SCT F11 (Celestron) telescope, using the eShell cross-dispersed echelle spectrograph (Shelyak) and a CCD camera ATIK 460EX with ICX694 (Sony) sensor.

(ix) At the Durtal Observatory with a $0.51 \mathrm{~m} \mathrm{~F} / 5$ telescope, using the eShel model of the echelle spectrograph (Shelyak); the detector was a KAF-3200ME CCD camera.

(x) At the Mirranook Observatory with a $0.25 \mathrm{~m} \mathrm{F6.4}$ SCT (Celestron) telescope, using a LISA spectrograph (Shelyak); the detector was an ATIK 314L CCD camera with a $23 \mu \mathrm{m}$ slit.

(xi) At the private station of the Sociéte Astronomique de Bourgogne-Dijon with a $0.2 \mathrm{~m}$ telescope (Celestron) using a LISA spectrograph (Shelyak); the detector was an ATIK 314L CCD camera.

(xii) At the private stations, Manhattan (Illinois) and Grand Lake (Colorado) by a $0.25 \mathrm{~m} \mathrm{LX200} \mathrm{SCT} \mathrm{telescope} \mathrm{us-}$ ing the Alpy 600 spectrograph (Shelyak); the detector was an ATIK 314L CCD camera.

For the purpose of modelling the SED we used low-resolution spectra $(R \sim 500-1000$, Table 1$)$, while the medium-resolution spectra $(R \sim 10000$, Table 2$)$ served to analyse variations in the line profiles. Relative flux units were converted into absolute fluxes with the aid of the (near-)simultaneous $B V R_{\mathrm{C}} I_{\mathrm{C}}$ photometry of Munari et al. (2013a) and/or $U B V$ photometry from the AAVSO database. Magnitudes were converted to fluxes according to the calibration of Henden \& Kaitchuck (1982). Observations were dereddened with $E_{B-V}=0.18$ (Munari et al. 2013b; Shore et al. 2013c; Chochol et al. 2014) by using the extinction curve of Cardelli et al. (1989). Then we scaled the dereddened spectra to the $V$ flux-point.

We selected only spectra with a good match at all available photometric flux-points, but especially in the $B$ band. The measured photometric magnitudes were corrected for emission lines to obtain fluxes of the true continuum (see Skopal 2007). Some spectra showed an excess or depression for $\lambda \lesssim 4500 \AA$. They were not used in our analysis, because the continuum profile sensitively depends on the effective temperature of the shell the primary parameter of our SED-fitting analysis (Sect. 3.1). 
Table 1. Log of low-resolution spectroscopic observations.

\begin{tabular}{|c|c|c|c|c|c|}
\hline $\begin{array}{l}\text { Day }^{a} \\
2013 \text { Aug. }\end{array}$ & $\begin{array}{l}\text { Julian date } \\
\text { JD } 24565 . .\end{array}$ & $\begin{array}{c}\text { Region } \\
{[\mathrm{nm}]}\end{array}$ & $\begin{array}{r}T_{\exp } \\
{[\mathrm{s}]} \\
\end{array}$ & $R^{b}$ & Observer \\
\hline 14.905 & 19.405 & $373-742$ & 623 & 754 & Guarro \\
\hline 14.934 & 19.434 & $373-743$ & 623 & 771 & Guarro \\
\hline 14.972 & 19.472 & $372-743$ & 528 & 755 & Guarro \\
\hline 15.004 & 19.504 & $371-743$ & 423 & 741 & Guarro \\
\hline 15.483 & 19.983 & $355-950$ & 300 & 500 & Fujii-san \\
\hline 15.496 & 19.996 & $380-730$ & 1778 & 1306 & Bohlsen \\
\hline 15.634 & 20.134 & $355-950$ & 300 & 500 & Fujii-san \\
\hline 15.804 & 20.304 & $333-758$ & 10 & 1000 & Tarasova \\
\hline 15.869 & 20.369 & $371-729$ & 791 & 675 & Terry \\
\hline 15.996 & 20.496 & $371-743$ & 370 & 784 & Guarro \\
\hline 16.198 & 20.698 & $372-757$ & 773 & 712 & Edlin \\
\hline 16.470 & 20.970 & $355-960$ & 300 & 500 & Fujii-san \\
\hline 16.512 & 21.012 & $355-960$ & 240 & 500 & Fujii-san \\
\hline 16.553 & 21.053 & $355-960$ & 240 & 500 & Fujii-san \\
\hline 16.595 & 21.095 & $355-960$ & 240 & 500 & Fujii-san \\
\hline 16.637 & 21.137 & $355-960$ & 220 & 500 & Fujii-san \\
\hline 16.740 & 21.240 & $355-960$ & 400 & 500 & Fujii-san \\
\hline 16.885 & 21.385 & $371-729$ & 3193 & 682 & Terry \\
\hline 16.950 & 21.450 & $379-722$ & 1914 & 912 & Favaro \\
\hline 17.134 & 21.634 & $375-700$ & 720 & 514 & Graham \\
\hline 17.265 & 21.765 & $375-740$ & 747 & 884 & Edlin \\
\hline 17.473 & 21.973 & $355-960$ & 400 & 500 & Fujii-san \\
\hline 17.543 & 22.043 & $355-960$ & 400 & 500 & Fujii-san \\
\hline 17.710 & 22.210 & $355-960$ & 400 & 500 & Fujii-san \\
\hline 17.898 & 22.398 & $361-738$ & 3854 & 513 & Antao \\
\hline 18.189 & 22.689 & $367-740$ & 2331 & 758 & Edlin \\
\hline 18.456 & 22.956 & $355-960$ & 280 & 500 & Fujii-san \\
\hline 18.520 & 23.020 & $355-960$ & 300 & 500 & Fujii-san \\
\hline 18.585 & 23.085 & $355-960$ & 220 & 500 & Fujii-san \\
\hline 18.873 & 23.373 & $365-735$ & 2061 & 513 & Antao \\
\hline 18.943 & 23.443 & $365-735$ & 2031 & 513 & Antao \\
\hline 19.151 & 23.651 & $375-739$ & 773 & 938 & Edlin \\
\hline 19.480 & 23.980 & $355-960$ & 260 & 500 & Fujii-san \\
\hline 19.550 & 24.050 & $355-960$ & 200 & 500 & Fujii-san \\
\hline 19.849 & 24.349 & $368-743$ & 327 & 760 & Guarro \\
\hline 19.887 & 24.387 & $333-757$ & 10 & 1000 & Tarasova \\
\hline 21.691 & 26.191 & $365-929$ & 91 & 500 & Fujii-san \\
\hline 21.822 & 26.322 & $368-735$ & 1367 & 591 & Thizy \\
\hline 26.161 & 30.661 & $360-740$ & 398 & 503 & Graham \\
\hline 28.477 & 32.977 & $355-960$ & 91 & 500 & Fujii-san \\
\hline \multicolumn{6}{|l|}{ Sept. } \\
\hline 13.536 & 49.036 & $355-960$ & 96 & 500 & Fujii-san \\
\hline 13.748 & 49.248 & $333-757$ & 10 & 1000 & Tarasova \\
\hline 20.542 & 56.042 & $355-960$ & 400 & 500 & Fujii-san \\
\hline 20.864 & 56.364 & $663-1020$ & 4310 & 1085 & Guarro \\
\hline
\end{tabular}

Notes. ${ }^{(a)}$ Mid of observation in UT; ${ }^{(b)}$ average resolution.

We also used near-IR JHKLM photometry of nova V339 Del as published by Burlak et al. (2013), Gehrz et al. (2013), Cass et al. (2013a,b) and Shenavrin et al. (2013). Resulting parameters were scaled to a distance of $3 \mathrm{kpc}$ (Chochol et al. 2014).

\section{Analysis and results}

The primary aim of this section is to match the observed SED in our spectra by an appropriate model, and in this way determine the physical parameters of the emitting region at a given time of the nova evolution.
Table 2. Log of medium-resolution spectroscopic observations.

\begin{tabular}{|c|c|c|c|c|c|}
\hline $\begin{array}{l}\text { Day }^{a} \\
\text { Aug. }\end{array}$ & $\begin{array}{c}\text { JD } \\
24565 . .\end{array}$ & $\begin{array}{c}\text { Region }^{b} \\
{[\mathrm{~nm}]}\end{array}$ & $\begin{array}{c}T_{\exp } \\
{[\mathrm{s}]} \\
\end{array}$ & $F_{\text {cont. }}{ }^{c}$ & Observer \\
\hline 14.844 & 19.344 & $418-732$ & 3636 & $9.7^{d}$ & Garde \\
\hline 14.885 & 19.385 & $418-732$ & 3349 & $10.0^{d}$ & Garde \\
\hline 14.985 & 19.485 & $\mathrm{H} \alpha$ & 3683 & 11.9 & Garde \\
\hline 15.027 & 19.527 & $\mathrm{H} \alpha$ & 3624 & 12.7 & Garde \\
\hline 15.062 & 19.562 & $\mathrm{H} \alpha$ & 2422 & 13.4 & Garde \\
\hline 15.850 & 20.350 & $\mathrm{H} \alpha$ & 3180 & 32.1 & Garde \\
\hline 15.885 & 20.385 & $\mathrm{H} \alpha$ & 2840 & 32.9 & Garde \\
\hline 15.885 & 20.385 & Raman & 2840 & 29.3 & Garde \\
\hline 16.910 & 21.410 & $\mathrm{H} \alpha$ & 3830 & 55.5 & Garde \\
\hline 16.910 & 21.410 & Raman & 3830 & 49.6 & Garde \\
\hline 17.842 & 22.342 & $\mathrm{H} \alpha$ & 4263 & 49.0 & Garde \\
\hline 17.967 & 22.467 & Raman & 4263 & 47.4 & WR13 $\mathrm{x}^{e}$ \\
\hline 18.044 & 22.544 & $\mathrm{H} \alpha$ & 3074 & 48.9 & Garde \\
\hline 18.913 & 23.413 & $\mathrm{H} \alpha$ & 622 & 48.7 & Lemoult \\
\hline 19.050 & 23.550 & Raman & 900 & 45.2 & WR13x \\
\hline 19.050 & 23.550 & $\mathrm{H} \alpha$ & 900 & 48.7 & WR13x \\
\hline 19.870 & 24.370 & $\mathrm{H} \alpha$ & 2877 & 49.0 & Lemoult \\
\hline 19.870 & 24.370 & Raman & 2877 & 47.0 & Lemoult \\
\hline 20.051 & 24.551 & $\mathrm{H} \alpha$ & 438 & 49.0 & WR13x \\
\hline 20.840 & 25.340 & Raman & 3659 & 34.7 & Garde \\
\hline 21.876 & 26.376 & Raman & 4027 & 25.5 & Buil \\
\hline 22.854 & 27.354 & Raman & 4575 & 21.5 & Garde \\
\hline 23.845 & 28.345 & Raman & 4921 & 19.0 & Garde \\
\hline 24.979 & 29.479 & Raman & 3618 & 17.1 & Buil \\
\hline 26.828 & 31.328 & Raman & 6040 & 13.1 & Garde \\
\hline 28.849 & 33.349 & $\mathrm{H} \alpha$ & 4546 & 13.9 & Buil \\
\hline \multicolumn{6}{|l|}{ Sept. } \\
\hline 01.881 & 37.381 & Raman & 2565 & 7.81 & Charbonne \\
\hline 05.955 & 41.455 & Raman & 827 & 6.02 & WR13x \\
\hline 10.840 & 46.340 & Raman & 2350 & 5.34 & Charbonne \\
\hline 21.825 & 57.325 & Raman & 6060 & 3.74 & Garde \\
\hline 22.858 & 58.358 & Raman & 2458 & 3.45 & Buil \\
\hline \multicolumn{6}{|l|}{ Oct. } \\
\hline 01.923 & 67.423 & Raman & 4555 & 1.10 & Buil \\
\hline
\end{tabular}

Notes. The average resolving power was $10000-11000 ;{ }^{(a)}$ mid of observation in UT; ${ }^{(b)}$ the used wavelength range; ${ }^{\left({ }^{c}\right)}$ the continuum flux at the $\mathrm{H} \alpha$ or the Raman $6825 \AA$ line in $10^{-12} \mathrm{erg} \mathrm{cm}^{-2} \mathrm{~s}^{-1} \AA^{-1}$; ${ }^{(d)} F_{\text {cont. }}$ at the $\mathrm{H} \alpha$ line; ${ }^{(e)}$ WR13x-collaboration team.

\subsection{Model SEDs}

During the fireball stage of novae, the radiation of their pseudophotospheres resembles that produced by a star of spectral type A to F (e.g. Warner 2008, Sect. 1 here). Accordingly, we compared the observed spectrum, $F(\lambda)$, with a synthetic one, $\mathcal{F}_{\lambda}\left(T_{\text {eff }}\right)$, i.e.

$$
F(\lambda)=\theta_{\mathrm{WD}}^{2} \mathcal{F}_{\lambda}\left(T_{\text {eff }}\right),
$$

where $\theta_{\mathrm{WD}}=R_{\mathrm{WD}} / d$ is the angular radius of the WD pseudophotosphere and $T_{\text {eff }}$ its effective temperature. Fitting parameters are $\theta_{\mathrm{WD}}$ and $T_{\mathrm{eff}}$, which define the effective radius of the shell as $R_{\mathrm{WD}}=\theta_{\mathrm{WD}} \times d$ and its luminosity $L_{\mathrm{WD}}=4 \pi d^{2} \theta_{\mathrm{WD}}^{2} T_{\mathrm{eff}}^{4}$ for the distance to the nova, $d$. A warm photospheric radiation of the nova was indicated already by our first spectrum from Aug. 14.844. The last spectrum showing unambiguously a dominant contribution from the warm stellar pseudophotosphere in the optical was observed on Aug. 19.887. Therefore, we associate this date with the end of the fireball stage of the nova V339 Del (see also Sect. 4.2.3).

In the SED-fitting analysis we compared a grid of synthetic models with the observed spectrum (1) and selected the one 
corresponding to a minimum of the reduced $\chi^{2}$ function. The continuum fluxes were estimated by eye. In this way we obtained the model variables $\theta_{\mathrm{WD}}$ and $T_{\text {eff. }}$. The grid of atmospheric models was prepared from that of Munari et al. (2005), for $T_{\text {eff }}=5000-15000 \mathrm{~K}$ with the step $\Delta T_{\text {eff }}=250 \mathrm{~K}$ and fixed other atmospheric parameters. The model resolution was accommodated to that of our spectra (see Skopal et al. 2011, for more details).

After the fireball stage, during the transition of the nova towards higher $T_{\text {eff }}$, its radiation ionizes the outer material, which reprocesses a fraction of it into the nebular emission. As a result, the nebular component of radiation starts to rival the stellar component from the WD pseudophotosphere in the optical/near-IR. Because of its higher $T_{\text {eff }}$, we observe only its long-wavelength tail in the optical, which can be approximated by a blackbody radiating at the temperature $T_{\mathrm{bb}}$. In this case, the observed spectrum can be expressed as

$F(\lambda)=\theta_{\mathrm{WD}}^{2} \pi B_{\lambda}\left(T_{\mathrm{bb}}\right)+k_{\mathrm{n}} \times \varepsilon_{\lambda}\left(T_{\mathrm{e}}\right)$,

where the factor $k_{\mathrm{n}}=E M / 4 \pi d^{2}$ scales the total volume emission coefficient $\varepsilon_{\lambda}\left(T_{\mathrm{e}}\right)$ of the nebular continuum to observations; $E M$ is the so-called emission measure. The variables determining the model SED (2) are $\theta_{\mathrm{WD}}, T_{\mathrm{bb}}, k_{\mathrm{n}}$ and $T_{\mathrm{e}}$. Equation (2) assumes that $T_{\mathrm{e}}$ and thus $\varepsilon_{\lambda}\left(T_{\mathrm{e}}\right)$ are constant throughout the nebula. For the sake of simplicity, we considered only the contribution of the hydrogen plasma (see e.g. Skopal 2014a, for more details). The first spectrum that could be matched by a superposition of just these two components of radiation was observed on Aug. 26.16.

Our spectra taken during the first few days after the fireball stage indicated a persisting contribution from the warm stellar pseudophotosphere. This is evident from the spectra of Aug. 20.194 (Edlin, ARAS), 20.463, 21.691 (Fujii $^{2}$ ), and 21.822 (Thizy, ARAS). They showed a steeper short-wavelength part of the spectrum for $\lambda \lesssim 5000 \AA$ and a flat continuum at a high level of a few times $10^{-11} \mathrm{erg} \mathrm{cm}^{-2} \mathrm{~s}^{-1} \AA^{-1}$ for $5000 \lesssim \lambda \lesssim 8000 \AA$. The former indicates the presence of a hotter stellar component of radiation, while the latter is given by superposition of a nebular component and that from the warm pseudophotosphere. Therefore, we matched the observed SED in this transition phase by radiation components according to Eq. (2) supplemented by a synthetic spectrum $\mathcal{F}_{\lambda}\left(T_{\text {eff }}\right)$ for $T_{\text {eff }}=5000-6000 \mathrm{~K}$ (see Sect. 3.2.2).

Modelling the SED after the fireball stage is only unambiguous if we use data covering a broad spectral region. We used the spectra of Aug. 22, 28, Sept. 13, and 20, which cover a more extended wavelength region and were obtained simultaneously with the photometric near-IR observations. The near-IR fluxes are especially important to recognize the dust contribution (see Fig. 2).

The resulting parameters are listed in Table 3, and examples of corresponding models fitting the selected spectra are depicted in Figs. 1 and 2, while Fig. 3 shows the evolution of the fundamental parameters, $L_{\mathrm{WD}}, R_{\mathrm{WD}}$ and $T_{\mathrm{eff}}$, during the fireball stage (Aug. 14.8-19.9, 2013). Although that only static atmospheric models were compared, the resulting models express the measured continuum well.

\subsubsection{Estimate of uncertainties}

In the range of $T_{\text {eff }}$ between $\sim 5000$ and $\sim 15000 \mathrm{~K}$, its value is sensitive to the overall profile of the measured continuum in the

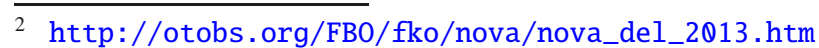

Table 3. Physical parameters of nova V339 Del.

\begin{tabular}{|c|c|c|c|c|}
\hline $\begin{array}{l}\text { Date } \\
\text { Aug. }\end{array}$ & $\begin{array}{l}T_{\text {eff }} \\
{[\mathrm{K}]}\end{array}$ & $\begin{array}{r}\theta_{\mathrm{WD}} \\
{\left[10^{-10}\right]} \\
\end{array}$ & $\begin{array}{l}R_{\mathrm{WD}} \\
{\left[R_{\odot}\right]}\end{array}$ & $\begin{array}{c}L_{\mathrm{WD}} \\
{\left[10^{38} \mathrm{erg} \mathrm{s}^{-1}\right]}\end{array}$ \\
\hline \multicolumn{5}{|c|}{ The fireball stage } \\
\hline 14.844 & $10000 \pm 400$ & $5.0 \pm 0.2$ & $66 \pm 2$ & $1.5 \pm 0.2$ \\
\hline 14.885 & $9750 \pm 250$ & $5.2 \pm 0.2$ & $68 \pm 2$ & $1.5 \pm 0.1$ \\
\hline 14.905 & $9500 \pm 250$ & $5.8 \pm 0.2$ & $77 \pm 2$ & $1.3 \pm 0.1$ \\
\hline 14.934 & $9500 \pm 250$ & $5.7 \pm 0.2$ & $76 \pm 2$ & $1.6 \pm 0.1$ \\
\hline 14.972 & $9000 \pm 250$ & $6.2 \pm 0.2$ & $82 \pm 3$ & $1.5 \pm 0.2$ \\
\hline 15.004 & $9000 \pm 250$ & $6.3 \pm 0.2$ & $84 \pm 3$ & $1.6 \pm 0.2$ \\
\hline 15.483 & $10000 \pm 250$ & $7.3 \pm 0.4$ & $97 \pm 5$ & $3.2 \pm 0.4$ \\
\hline 15.496 & $10000 \pm 125$ & $7.3 \pm 0.3$ & $96 \pm 4$ & $3.2 \pm 0.2$ \\
\hline 15.634 & $10000 \pm 250$ & $7.7 \pm 0.4$ & $103 \pm 5$ & $3.7 \pm 0.4$ \\
\hline 15.804 & $12000 \pm 500$ & $6.6 \pm 0.3$ & $88 \pm 4$ & $5.6 \pm 0.7$ \\
\hline 15.869 & $11500 \pm 250$ & $7.7 \pm 0.3$ & $102 \pm 4$ & $6.3 \pm 0.5$ \\
\hline 15.996 & $12000 \pm 500$ & $8.3 \pm 0.3$ & $110 \pm 4$ & $8.6 \pm 0.8$ \\
\hline 16.198 & $9500 \pm 125$ & $12.1 \pm 0.5$ & $161 \pm 7$ & $7.3 \pm 0.5$ \\
\hline 16.470 & $8500 \pm 125$ & $15.5 \pm 0.5$ & $205 \pm 7$ & $7.6 \pm 0.5$ \\
\hline 16.512 & $8500 \pm 250$ & $15.6 \pm 0.7$ & $207 \pm 9$ & $7.8 \pm 0.7$ \\
\hline 16.553 & $8500 \pm 250$ & $15.6 \pm 0.7$ & $207 \pm 9$ & $7.8 \pm 0.7$ \\
\hline 16.595 & $8625 \pm 125$ & $14.6 \pm 0.5$ & $195 \pm 7$ & $7.2 \pm 0.4$ \\
\hline 16.637 & $8000 \pm 250$ & $16.7 \pm 0.8$ & $222 \pm 11$ & $7.0 \pm 0.7$ \\
\hline 16.740 & $8000 \pm 250$ & $16.3 \pm 0.8$ & $217 \pm 11$ & $6.6 \pm 0.7$ \\
\hline 16.885 & $7500 \pm 250$ & $17.2 \pm 0.8$ & $229 \pm 11$ & $5.7 \pm 0.7$ \\
\hline 16.950 & $7500 \pm 250$ & $17.2 \pm 0.8$ & $229 \pm 11$ & $5.7 \pm 0.7$ \\
\hline 17.134 & $7750 \pm 250$ & $15.3 \pm 0.7$ & $204 \pm 9$ & $5.2 \pm 0.6$ \\
\hline 17.265 & $8000 \pm 125$ & $14.4 \pm 0.4$ & $192 \pm 5$ & $5.2 \pm 0.4$ \\
\hline 17.473 & $7500 \pm 250$ & $16.1 \pm 0.7$ & $214 \pm 9$ & $5.0 \pm 0.5$ \\
\hline 17.543 & $7250 \pm 250$ & $17.5 \pm 0.8$ & $233 \pm 11$ & $5.2 \pm 0.6$ \\
\hline 17.710 & $7125 \pm 125$ & $18.3 \pm 0.7$ & $243 \pm 9$ & $5.3 \pm 0.3$ \\
\hline 17.898 & $7250 \pm 250$ & $17.8 \pm 0.8$ & $236 \pm 11$ & $5.3 \pm 0.6$ \\
\hline 18.189 & $7500 \pm 250$ & $16.7 \pm 0.7$ & $222 \pm 9$ & $5.4 \pm 0.5$ \\
\hline 18.456 & $7125 \pm 125$ & $19.1 \pm 0.7$ & $254 \pm 9$ & $5.8 \pm 0.3$ \\
\hline 18.520 & $7250 \pm 250$ & $18.5 \pm 0.8$ & $245 \pm 11$ & $5.7 \pm 0.6$ \\
\hline 18.585 & $7375 \pm 125$ & $17.7 \pm 0.7$ & $236 \pm 9$ & $5.6 \pm 0.3$ \\
\hline 18.873 & $7250 \pm 250$ & $17.4 \pm 0.9$ & $231 \pm 12$ & $5.1 \pm 0.6$ \\
\hline 18.943 & $7250 \pm 250$ & $17.6 \pm 0.9$ & $234 \pm 12$ & $5.2 \pm 0.6$ \\
\hline 19.151 & $7250 \pm 250$ & $17.4 \pm 0.9$ & $232 \pm 12$ & $5.1 \pm 0.6$ \\
\hline 19.480 & $6500 \pm 250$ & $20.9 \pm 0.9$ & $278 \pm 12$ & $4.8 \pm 0.6$ \\
\hline 19.550 & $6500 \pm 250$ & $20.3 \pm 0.9$ & $270 \pm 12$ & $4.5 \pm 0.6$ \\
\hline 19.849 & $6250 \pm 250$ & $21.7 \pm 1.0$ & $289 \pm 13$ & $4.4 \pm 0.7$ \\
\hline 19.887 & $6000 \pm 250$ & $23.9 \pm 1.2$ & $318 \pm 16$ & $4.5 \pm 0.8$ \\
\hline \multicolumn{5}{|c|}{ Transition to a harder spectrum } \\
\hline $21.691^{a}$ & $>37000$ & $<1.4$ & $<19$ & $>22$ \\
\hline $28.477^{b}$ & $>43000$ & $<1.0$ & $<13$ & $>21$ \\
\hline Sept. & & & & \\
\hline $13.642^{c}$ & $>100000$ & $<0.12$ & $<1.6$ & $>8.8$ \\
\hline $20.703^{d}$ & $>150000$ & $<0.046$ & $<0.6$ & $>6.5$ \\
\hline
\end{tabular}

Notes. Nebular component: ${ }^{(a)} T_{\mathrm{e}}=(2.0 \pm 0.5) \times 10^{4} \mathrm{~K}, E M=(2.0 \pm$ $0.2) \times 10^{62} \mathrm{~cm}^{-3} ;{ }^{(b)} T_{\mathrm{e}}=(1.5 \pm 0.2) \times 10^{4} \mathrm{~K}, E M=(1.8 \pm 0.1) \times$ $10^{62} \mathrm{~cm}^{-3} ;{ }^{(c)} T_{\mathrm{e}}=(4 \pm 0.5) \times 10^{4} \mathrm{~K}, E M=(1.4 \pm 0.1) \times 10^{62} \mathrm{~cm}^{-3}$; ${ }^{(d)} T_{\mathrm{e}}=(5 \pm 0.5) \times 10^{4} \mathrm{~K}, E M=(1.0 \pm 0.1) \times 10^{62} \mathrm{~cm}^{-3}$, dust component: $T_{\text {dust }}=(1350 \pm 50) \mathrm{K}, L_{\text {dust }}=(1.1 \pm 0.2) \times 10^{37} \mathrm{erg} \mathrm{s}^{-1}$.

optical. This allowed us to estimate $T_{\text {eff }}$ with a relative uncertainty of $<4 \%$. For the selected spectra (Sect. 2), $\Delta T_{\text {eff }}$ was well within the temperature step in the used grid of models, that is, between 250 and $500 \mathrm{~K}$ for $T_{\text {eff }}<10000$ and $>10000 \mathrm{~K}$. In some cases it was possible to estimate $\Delta T_{\text {eff }}$ to $\sim 125 \mathrm{~K}$ by interpolating the neighbouring models around the measured spectrum (Table 3).

The uncertainty of the scaling factor, $\theta_{\mathrm{WD}}$, is primarily given by the accuracy of the measured continuum, which was estimated to be $<3 \%$ for the used spectra. Different $T_{\text {eff }}$ requires 
A. Skopal et al.: Early evolution of the extraordinary Nova Delphini 2013 (V339 Del)
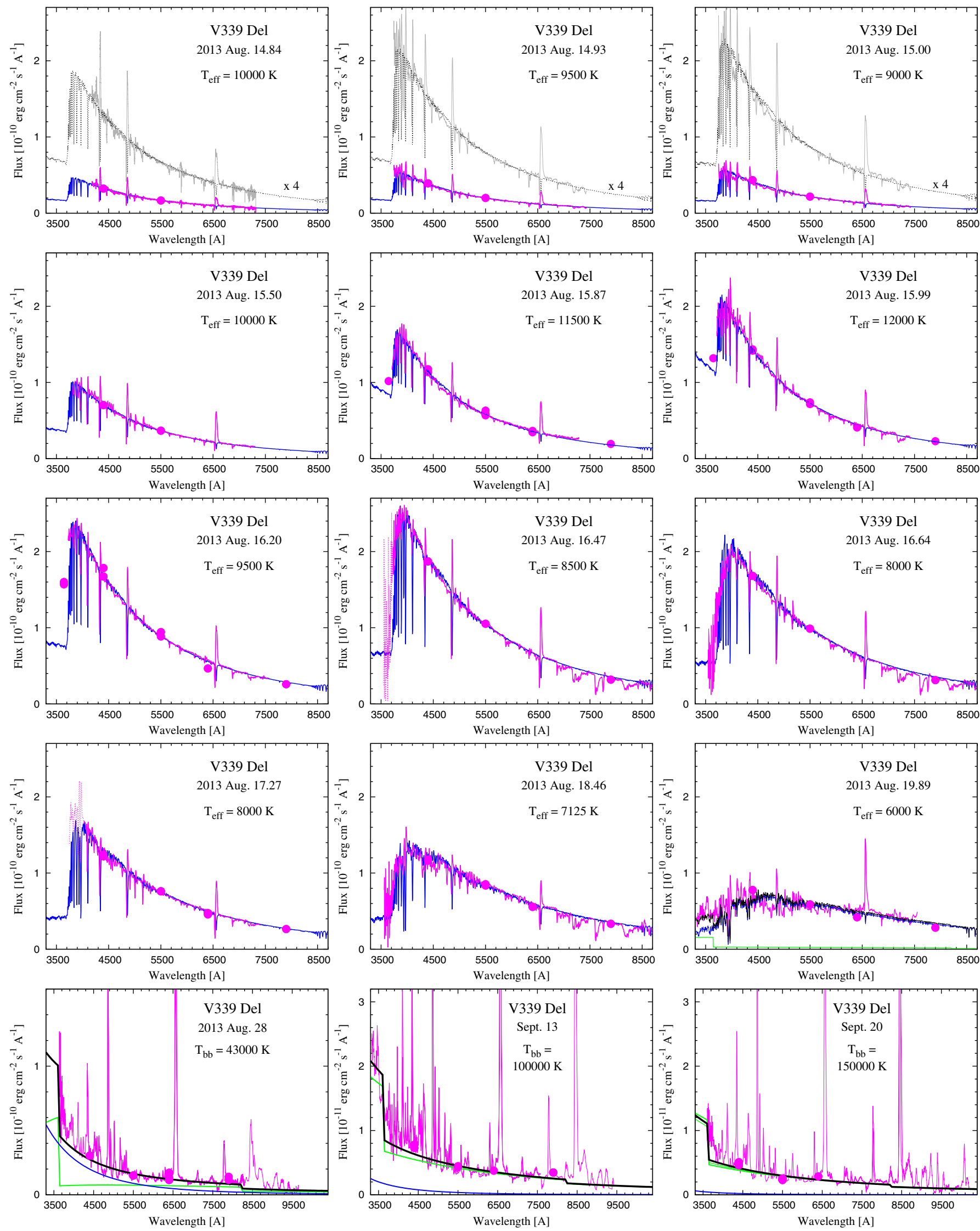

Fig. 1. Comparison of the observed (in magenta: spectrum and photometric flux-points) and model SEDs of V339 Del. During the fireball stage (2013, Aug. 14.84 to 19.89), the model SED is represented by a synthetic spectrum (blue line, Eq. (1)), while during the following stage with a harder spectrum (bottom row of panels) the model SED (black line) is given by a superposition of the radiation from the WD pseudophotosphere (blue line) and the nebula (green line) according to Eq. (2). 


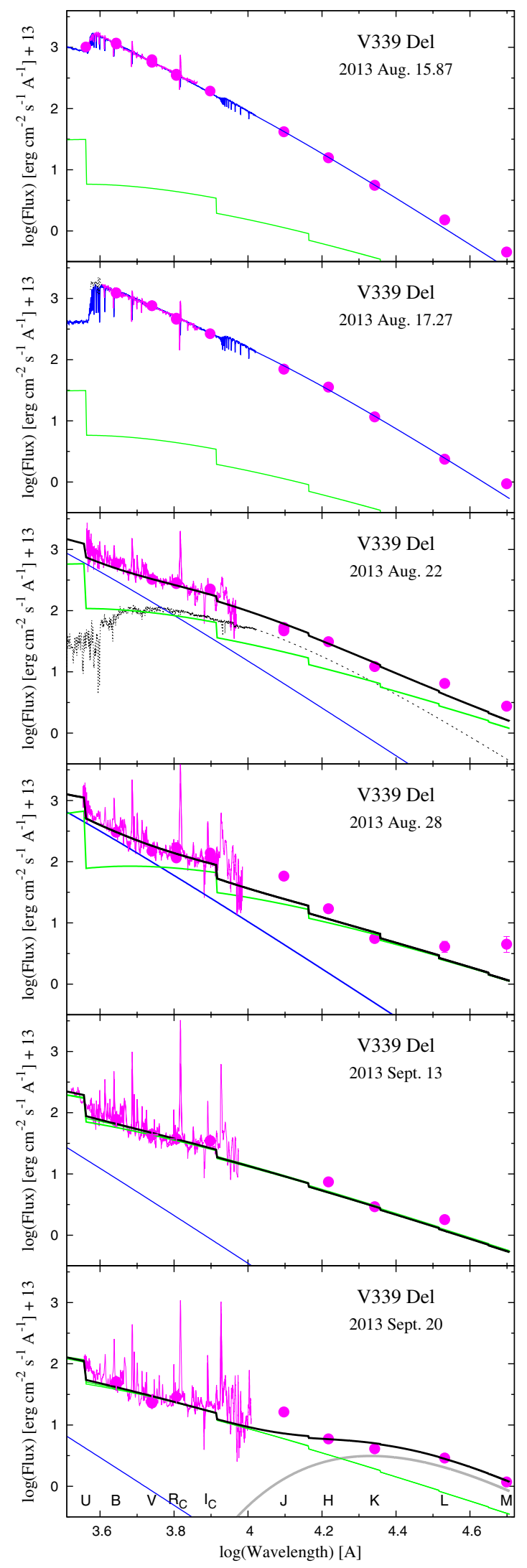

Fig. 2. Optical/near-IR SEDs of nova V339 Del during the fireball stage and beyond. Denotation of lines and points is the same as in Fig. 1. In addition, the dotted line in the third panel from the top represents a remnant of the WD shell and the grey line in the bottom panel denotes the dust component (Sect. 3.2.2). The nebular continuum during the fireball stage (top two panels) was only roughly estimated from the $\mathrm{H} \alpha$ flux for comparison.
August 2013 (UT)

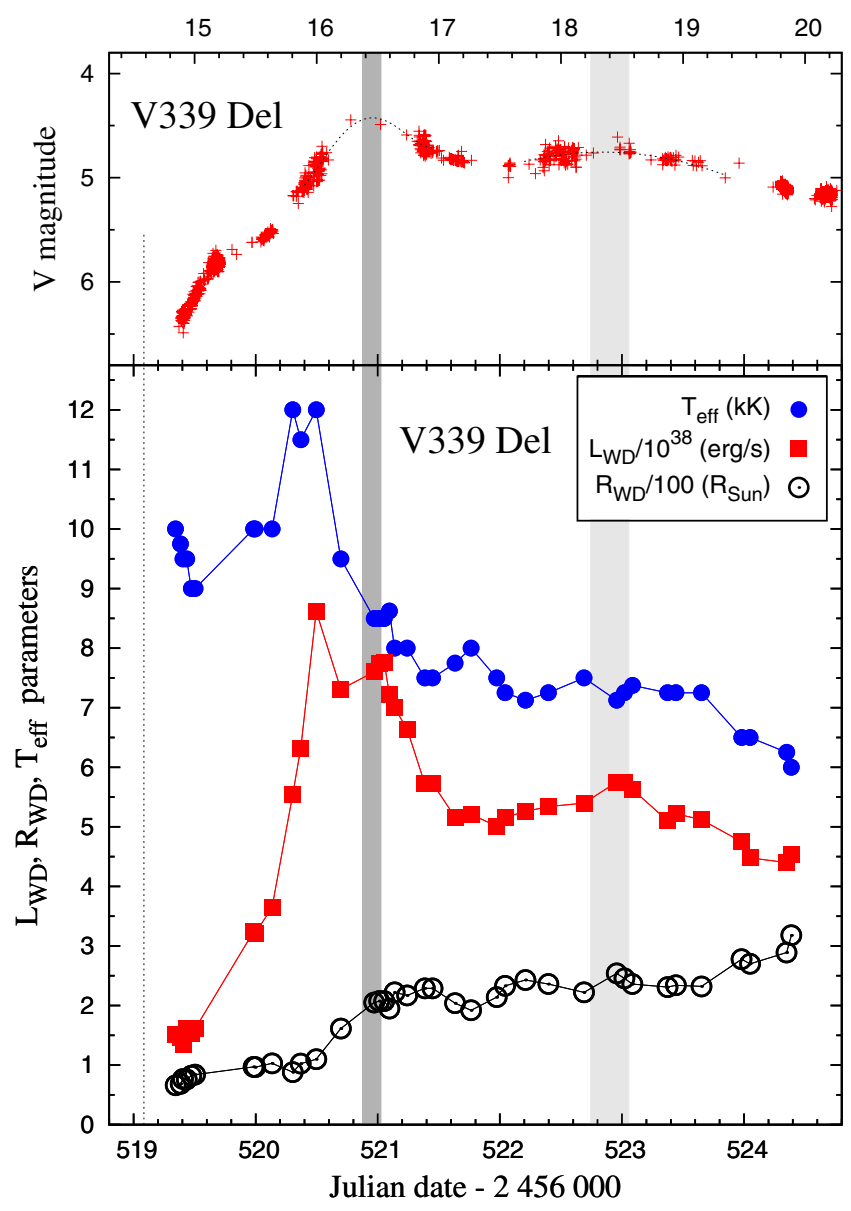

Fig. 3. Evolution of the parameters $L_{\mathrm{WD}}, R_{\mathrm{WD}}$, and $T_{\mathrm{eff}}$ of nova V339 Del during its fireball stage. The dotted line and grey belts mark the times of the nova discovery and the maxima in the $V$-light curve (Aug. $16.45 \pm 0.06,18.4 \pm 0.11$ ). Data are listed in Table 3 .

different scaling. $\Delta T_{\text {eff }}$ of 250 to $500 \mathrm{~K}$ corresponds to relative errors in $\theta_{\mathrm{WD}}$ of $2 \%$ to $4 \%$. Thus, the largest relative errors in $\theta_{\mathrm{WD}}$ range between 5 and $7 \%$.

The uncertainty in $L_{\mathrm{WD}}$ was determined as the root mean square error using the total differential of the function $L_{\mathrm{WD}}=$ $4 \pi d^{2} \theta_{\mathrm{WD}}^{2} T_{\mathrm{eff}}^{4}$ for given uncertainties in $T_{\mathrm{eff}}$ and $\theta_{\mathrm{WD}}$. The relative error of $L_{\mathrm{WD}}$ was found to be around $10 \%$, depending mainly on the uncertainty in $T_{\text {eff }}$ (see Table 3 ).

\subsection{Evolution of fundamental parameters}

\subsubsection{Fireball stage}

Following Fig. 3, we describe the temporal evolution of $T_{\text {eff }}$, $R_{\mathrm{WD}}$, and $L_{\mathrm{WD}}$ of the WD pseudophotosphere (also called the envelope or shell) during its fireball stage as follows:

Already a few hours after the discovery, the radiative output of the nova dominated the optical. A comparison of synthetic models with the observed spectra (Eq. (1), Figs. 1 and 3) showed that the nova envelope was radiating at $T_{\text {eff }}=$ $6000-12000 \mathrm{~K}$ during its fireball stage (Table 3). Our first spectra from Aug. 14.8-15.0, taken around 1.5 days prior to the photometric $V$-band maximum, suggested a transient rapid decrease in $T_{\text {eff }}$ from $\sim 10000 \mathrm{~K}$ to $\sim 9000 \mathrm{~K}$ (see the top row of Fig. 1) and a gradual increase of $R_{\mathrm{WD}}$ from $\sim 66$ to $\sim 84 R_{\odot}$ at a constant $L_{\mathrm{WD}}$ of $\sim 1.5 \times 10^{38} \mathrm{erg} \mathrm{s}^{-1}$. 
About half a day later ( Aug. 15.5), during the first short plateau phase in the light curve $(V \sim 5.6)$, the continuum profile of our spectra reflected an unambiguous increase in the temperature to $T_{\text {eff }}=10000 \pm 250 \mathrm{~K}$, but at nearly unchanged radius, $\$ 100 R_{\odot}$, implying an increase of $L_{\mathrm{WD}}$ by a factor of $\gtrsim 2$. The following heating of the envelope to its maximum of $12000 \pm$ $500 \mathrm{~K}$ on Aug. 15.8-16.0, with only a small increase of its radius to $\approx 100(d / 3 \mathrm{kpc}) R_{\odot}$, implied a rapid increase of $L_{\mathrm{WD}}$ with a maximum of $\sim 8.6 \times 10^{38}(d / 3 \mathrm{kpc})^{2} \mathrm{erg} \mathrm{s}^{-1}$ on Aug. 16.0 (see the second row from the top of Fig. 1).

A rapid cooling of the envelope to $\sim 7500 \mathrm{~K}$ on Aug. 16.9 (i.e. at a rate of $\sim 210 \mathrm{~K} / \mathrm{h}$ ) was a result of the shell expansion by a factor of $\sim 2$, to $\sim 200(d / 3 \mathrm{kpc}) R_{\odot}$, which was followed by a general decrease of its luminosity to $\sim 5 \times 10^{38}(d / 3 \mathrm{kpc})^{2} \mathrm{erg} \mathrm{s}^{-1}$. At the $V$-brightness maximum (Aug. 16.45, see narrower grey belt in Fig. 3), we indicate a local maximum of the luminosity.

From Aug. 16.9 to the end of the fireball stage of the nova, $T_{\text {eff }}$ was gradually declining to $\sim 6000 \mathrm{~K}$, followed by a gradual increase of its radius up to $\approx 300(d / 3 \mathrm{kpc}) R_{\odot}$, while the corresponding luminosity denoted only a small decrease to $\sim 4.6 \times 10^{38}(d / 3 \mathrm{kpc})^{2} \mathrm{erg} \mathrm{s}^{-1}$. Our observations caught an interesting behaviour around a shallow secondary $V$-band maximum (Aug. 18.4, see broad grey belt in Fig. 3) corresponding to a small local maximum of $L_{\mathrm{WD}}$.

\subsubsection{Evolution beyond the fireball stage}

Two days after the end of the fireball stage, on Aug. 22 (day 5.5), the high level of the optical continuum for $\lambda>5000 \AA$ (Sect. 3.1) and the bright JHKLM magnitudes (Cass et al. 2013a) required the presence of a strong nebular radiation component with $E M \sim$ $2 \times 10^{62}(d / 3 \mathrm{kpc})^{2} \mathrm{~cm}^{-3}$ supplemented by a contribution from a $\sim 5000 \mathrm{~K}$ warm residual pseudophotosphere scaled to $\sim 9 \times$ $10^{37}\left(d / 3 \mathrm{kpc}^{2} \mathrm{erg} \mathrm{s}^{-1}\right.$ (dotted line in Fig. 2). The very high value of EM constrained the minimum of $T_{\mathrm{bb}} \sim 37000 \mathrm{~K}$ and $L_{\mathrm{WD}} \sim 2.2 \times 10^{39}(\mathrm{~d} / 3 \mathrm{kpc})^{2} \mathrm{erg} \mathrm{s}^{-1}$ to fit the short-wavelength part of the spectrum (for details see Appendix A of Skopal $2014 \mathrm{~b}$ ). The corresponding radius of the WD photosphere was $R_{\mathrm{WD}} \lesssim 19(d / 3 \mathrm{kpc}) R_{\odot}$.

On Aug. 28.5 (day 12), the warm stellar pseudophotosphere could no longer be identified. The spectrum for $\lambda>6000 \AA$ was dominated solely by the nebular continuum with $E M=$ $1.8 \times 10^{62}(d / 3 \mathrm{kpc})^{2} \mathrm{~cm}^{-3}$. As in the previous case, the stellar component of radiation from the WD photosphere was determined such that its Lyman-continuum photons are just capable of giving rise to the measured EM and the component fits the short-wavelength part of the spectrum. In this way, we obtained $T_{\mathrm{bb}} \gtrsim 43000 \mathrm{~K}, R_{\mathrm{WD}} \lesssim 13(d / 3 \mathrm{kpc}) R_{\odot}$ and $L_{\mathrm{WD}} \gtrsim$ $2.1 \times 10^{39}\left(d / 3 \mathrm{kpc}^{2} \mathrm{erg} \mathrm{s}^{-1}\right.$.

On Sept. 13 (day 28), the nebular component of radiation entirely dominated the optical to near-IR $(330 \mathrm{~nm}-3.4 \mu \mathrm{m})$. A contribution from the WD photosphere could not be recognized in the optical spectrum (see Figs. 1 and 2). The slope of the continuum and a relatively small Balmer jump in emission corresponded to a high $T_{\mathrm{e}}$. To match the observed continuum we scaled the $\varepsilon_{\lambda}\left(T_{\mathrm{e}}=40000 \mathrm{~K}\right)$ coefficient with $k_{\mathrm{n}}=$ $1.3 \times 10^{17} \mathrm{~cm}^{-5}$, i.e. $E M=1.4 \times 10^{62}(d / 3 \mathrm{kpc})^{2} \mathrm{~cm}^{-3}$ (Eq. (2)). Because no observations in the UV are available, we can only estimate the lower limit of the WD luminosity and temperature. There are two principal constraints:

(i) The WD contribution cannot considerably influence even the short-wavelength part of the optical spectrum because this would lower the nebular contribution, the amount of which in the near-IR is justified by the $H K L$ fluxes.

(ii) The high EM requires a high rate of hydrogen-ionizing photons, $L_{\mathrm{ph}}=\alpha_{\mathrm{B}}\left(T_{\mathrm{e}}\right) \times E M \sim 1.4 \times 10^{49} \mathrm{~s}^{-1}$ for the recombination coefficient $\alpha_{\mathrm{B}}\left(T_{\mathrm{e}} \geq 30000 \mathrm{~K}\right) \sim 1 \times 10^{-13} \mathrm{~cm}^{3} \mathrm{~s}^{-1}$ (e.g. Péquignot et al. 1991). These conditions thus require the radiation at $T_{\mathrm{bb}}>100000 \mathrm{~K}$ scaled with $\theta_{\mathrm{WD}}<1.2 \times 10^{-11}$ (i.e. $R_{\mathrm{WD}}<1.6 R_{\odot}$ ), which correspond to $L_{\mathrm{WD}}>8.8 \times$ $10^{38} \mathrm{erg} \mathrm{s}^{-1}$.

On Sept. 20 (day 35), the continuum profile remained nearly unchanged, but with a flux level by a factor of $\sim 1.7$ lower than on Sept. 13 (see Fig. 1). Taking into account the same conditions as mentioned above, the model SED corresponded to $T_{\mathrm{e}} \sim$ $50000 \mathrm{~K}, E M=1.0 \times 10^{62}(d / 3 \mathrm{kpc})^{2} \mathrm{~cm}^{-3}$, which constrains $T_{\mathrm{bb}}>150000 \mathrm{~K}, R_{\mathrm{WD}}<0.6 R_{\odot}$ and $L_{\mathrm{WD}}>6.5 \times 10^{38} \mathrm{erg} \mathrm{s}^{-1}$. However, the near-IR HKLM flux-points from Sept. 21.8 were located clearly above the nebular continuum (see Fig. 2), indicating the presence of a dust component in the spectrum. Our model SED thus confirms the dust formation reported by Shenavrin et al. (2013) and suggests that it occurred already around Sept. 21 (see also Cass et al. 2013c). We matched the dust component with a blackbody temperature $T_{\text {dust }}=1350 \pm 50 \mathrm{~K}$ and a luminosity $L_{\text {dust }}=(1.1 \pm 0.2) \times 10^{37} \mathrm{erg} \mathrm{s}^{-1}$.

Model SEDs during the post-fireball stage are plotted in Fig. 2. One of the most interesting results of modelling the SED during this period is the persistent super-Eddington luminosity, which appeared to be even higher than during the fireball phase (see Table 3). The same effect was found for RS Oph by Skopal (2014b).

\subsection{Evolution of the $\mathrm{H} \alpha$ line profile}

Figure 4 shows the evolution of the $\mathrm{H} \alpha$ line during the fireball stage. Its profile was of P Cygni-type. To separate its main components, we fitted the profile with three Gaussian functions, two for its emission component and one to match the absorption component (dotted lines in Fig. 4). The main characteristics of the temporal evolution of the $\mathrm{H} \alpha$ profile can be summarized as follows:

(i) A strong absorption component was always present. Its radial velocity (RV) was gradually decreasing from $\sim-1600 \mathrm{~km} \mathrm{~s}^{-1}$, as was measured on our first spectrum from Aug. 14.84, to $\sim-730 \mathrm{~km} \mathrm{~s}^{-1}$, at the maximum of the envelope inflation, around Aug. 19.87 (see panel a of Fig. 7). The absorbed flux was gradually increasing until Aug. 18, when its amount was by a factor of $\sim 8$ higher than measured on our first spectra (see panel $b$ of the figure). It is of interest to note that the absorption component was strong enough to cut parts of the emission component at its blue side even after the fireball stage (¿Aug. 20.0, see Fig. 8).

(ii) Prior to the optical maximum, the emission part of the profile was flat-topped, suggesting that it was composed of two strongly blended components. After the $V$-maximum, the $\mathrm{H} \alpha$ emission narrowed, mainly at the blue side, and its core became single-peaked. Around Aug. 18, when the absorbed flux reached its maximum, a transient decrease in the $\mathrm{H} \alpha$ emission was observed (panel c of Fig. 7).

(iii) At the end of the fireball phase, $\gtrsim$ Aug. 18.9, the red emission wing expanding to $R V \sim+2750 \mathrm{~km} \mathrm{~s}^{-1}$ increased. Its blue counterpart extended to $R V \sim-2550 \mathrm{~km} \mathrm{~s}^{-1}$ since Aug. 19.87. These values were almost unchanged during 


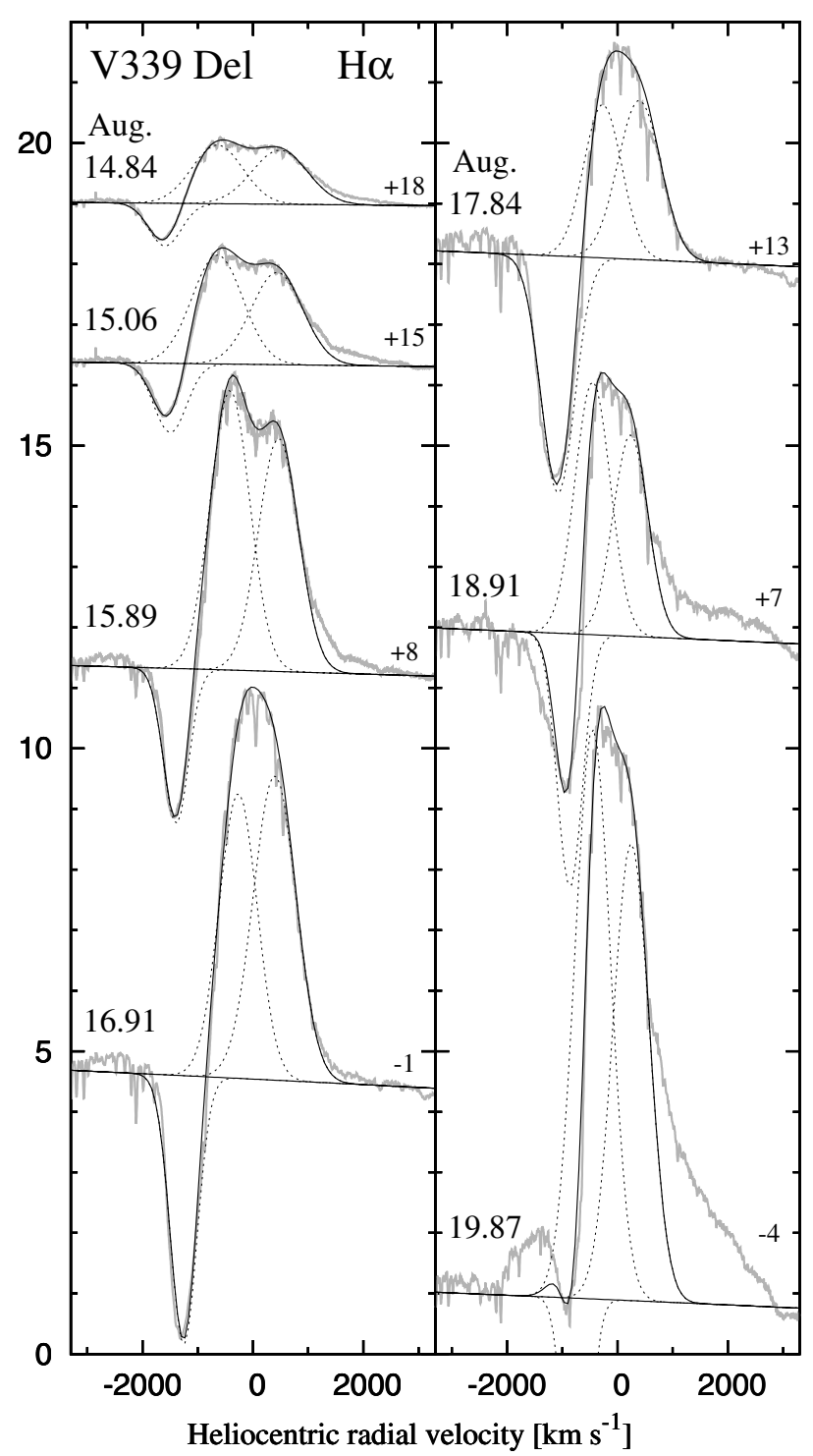

Fig. 4. Evolution of the $\mathrm{H} \alpha$ line profile during the fireball phase of V339 Del (grey lines). Each profile was fitted with three Gaussian functions (dotted curves) to isolate its main components (Sect. 3.3). Numbers on the right side mark the shift of the profile in the given units for a better visualization. Dates are marked at the left side. Fluxes are in $10^{-11} \mathrm{erg} \mathrm{cm}^{-2} \mathrm{~s}^{-1} \AA^{-1}$.

the investigated period of the nova evolution. Some examples are shown in Fig. 8. Accordingly, we adopted the terminal velocity of the broad wings as their average, i.e. $v_{\infty}=2650 \pm 100 \mathrm{~km} \mathrm{~s}^{-1}$.

\subsection{Raman-scattered O VI $1032 \AA$ line - first time seen in a classical nova}

A transient emergence of the Raman-scattered O VI $1032 \AA$ line in the spectrum of V339 Del was very startling, because its induced faint, broad emission feature around $\lambda 6830 \AA$ has never been observed in the spectrum of a classical nova (see Skopal et al. 2014, for the first note).

Figure 5 shows the temporal evolution of this feature. It was observed in the spectrum of V339 Del for more than one month, from Aug. 19 to the end of the first plateau phase in the light curve around Sept. 23, 2013 (day 37). During the fireball stage, no Raman emission was detected because of the

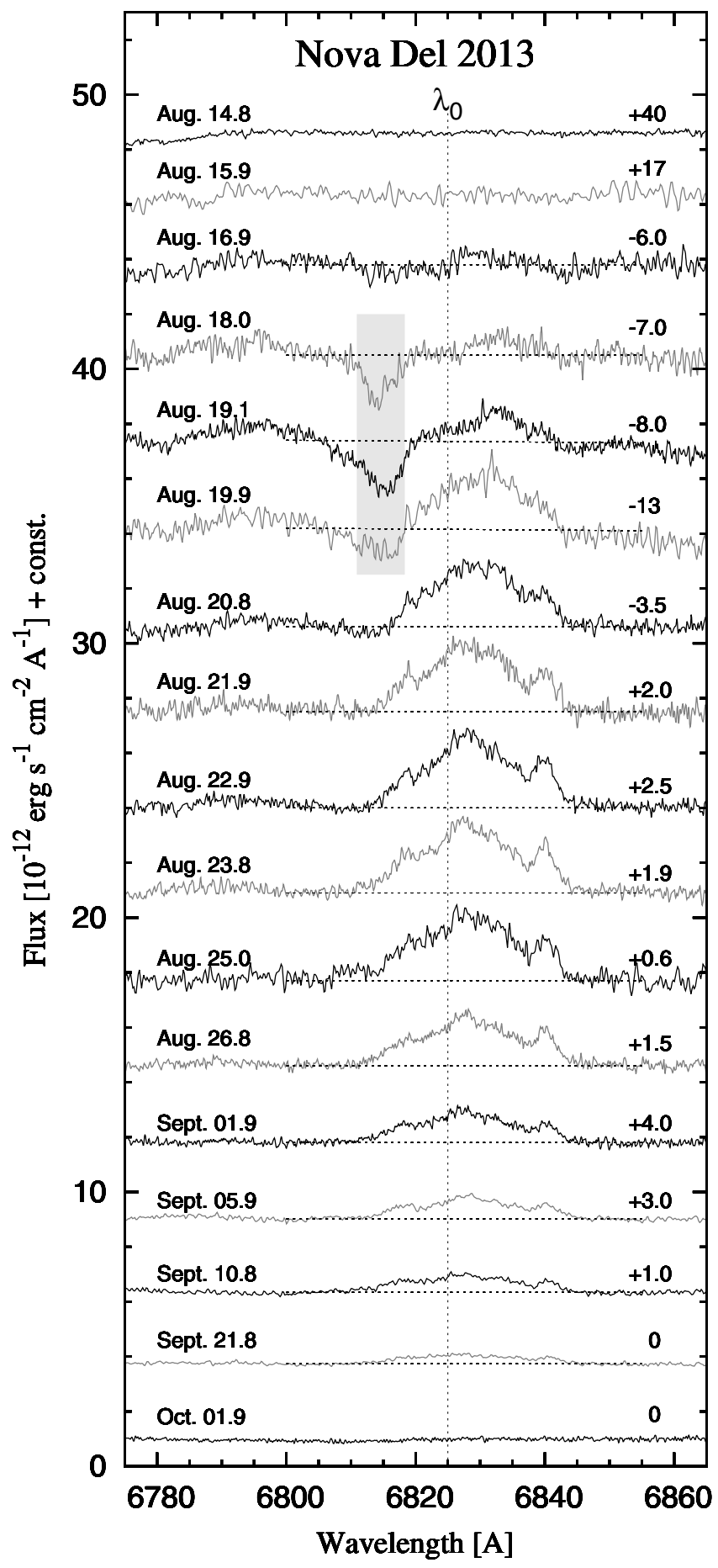

Fig. 5. Evolution of the Raman-scattered O VI $1032 \AA$ line becoming evident as an emission feature around $6830 \AA$ since the onset of the outburst on Aug. 14 to its disappearance. The grey belt marks an absorption that developed at the end of the fireball stage. Numbers on the right side mark the shift of the local continuum (dotted lines) with respect to the units of the flux scale (arbitrarily added for a better visualization). Observing dates are marked at the left side.

low temperature of the ionizing source, at which no original O VI photons could be created. From Aug. 20 on, the flux of the Raman line was decreasing and vanished completely by the end of September. Its $F W Z I$ was $\gtrsim 30 \AA$ and the profile showed a redward-shifted component around $6840 \AA$ and a faint blueshifted shoulder. A similar structure of the Raman-scattered line 


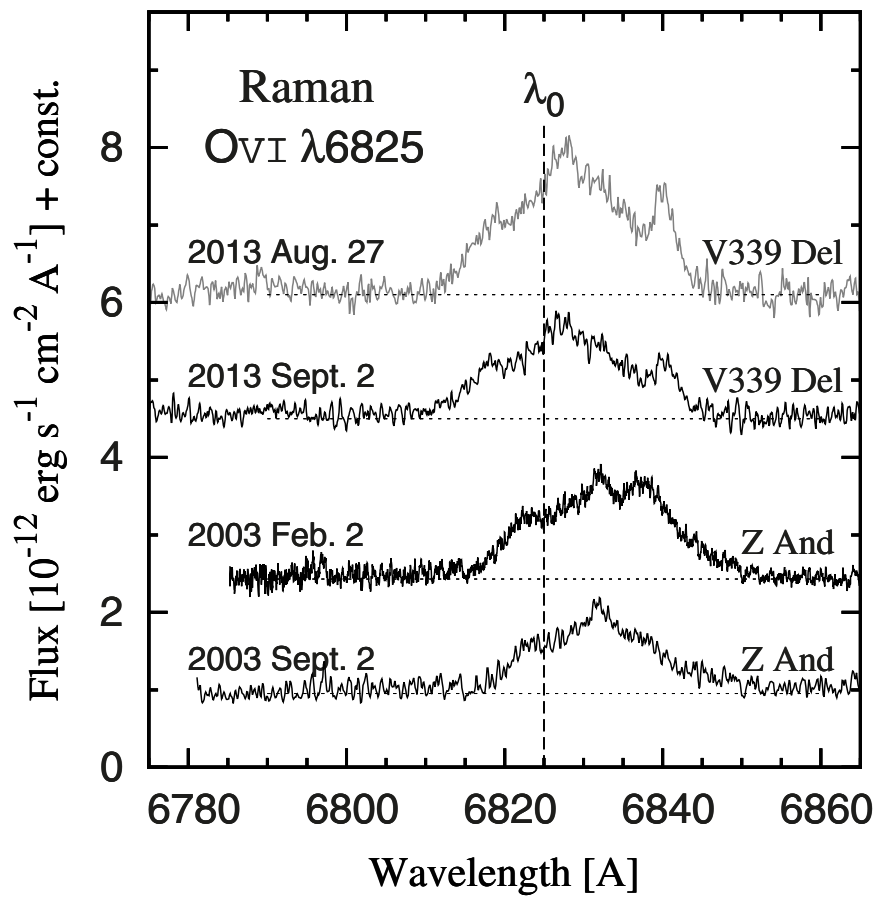

Fig. 6. Comparison of the Raman $6825 \AA$ lines from the spectrum of V339 Del (the top two profiles) with those observed in the spectrum of the classical symbiotic star Z And at the end of its 2000-03 active phase (adapted from Skopal et al. 2006). The similarity of these profiles supports the presence of the Raman-scattering process in the spectrum of the classical nova V339 Del (Sects. 3.4 and 4.2.4).

profile has frequently been recognized in the spectra of symbiotic stars (see Fig. 6). Energy conservation of the Raman process implies the broadening of the scattered line with a factor of $\left(\lambda_{\text {Ram }} / \lambda_{\text {OVI }}\right)^{2} \sim 44$ (Nussbaumer et al. 1989). Thus, the $F W Z I(6830) \approx 30 \AA$ corresponds to a maximum broadening of the original $1032 \AA$ line of $\approx 0.7 \AA$, i.e. $\approx 200 \mathrm{~km} \mathrm{~s}^{-1}$, which corresponds to expected movements of emitting material in the vicinity of the hot WD. Since October 1, just after the first plateau phase of the light curve, when a significant X-ray flux was first reported (Page et al. 2013), the Raman line could no longer been discerned in our spectra.

The appearance of the Raman-scattered line during a classical nova outburst is an unexpected important event that places strong constraints on the ionization structure of the nova ejecta (Sect. 4.2.4).

\section{Interpretation of observations}

\subsection{Evolution of the parameters $L_{\mathrm{WD}}, R_{\mathrm{WD}}$, and $T_{\mathrm{eff}}$}

The comprehensive spectroscopic and photometric observations of nova V339 Del, performed immediately after its discovery, allowed us to map in detail the evolution of its fundamental parameters even during the initial phase of its explosion. This showed, however, that the nova was not evolving strictly according to the canonical view, which assumes a gradual cooling and expanding of the nova envelope at a constant luminosity during the fireball stage (e.g. Shore et al. 1994; Schwarz et al. 2001).

The parameters $L_{\mathrm{WD}}, R_{\mathrm{WD}}$, and $T_{\mathrm{eff}}$ behaved as commonly expected only after the visual maximum, since Aug. 17 (Fig. 3, Sect. 3.2.1). The evolution of the nova prior to the $V$-maximum was unconventional. The transient increase in $T_{\text {eff }}$ from $\sim 9000$ (Aug. 14.97) to $\sim 12000 \mathrm{~K}$ (Aug. 15.9 ) at a nearly constant $R_{\mathrm{WD}}$ was caused by a rapid increase in the luminosity from $\sim 1.5$ to $\sim 8.6 \times 10^{38} \mathrm{erg} \mathrm{s}^{-1}$ (Fig. 3). At the maximum of $L_{\mathrm{WD}}$ and $T_{\mathrm{eff}}$, the effective radius $R_{\mathrm{WD}}$ started to expand rapidly. During about one day, from $\sim$ Aug. 16 to 17 , its value increased by a factor of $\gtrsim 2$, while $T_{\text {eff }}$ and $L_{\mathrm{WD}}$ decreased and followed the standard evolution at approximately constant, but super-Eddington luminosity $L_{\mathrm{WD}} \sim 5 \times 10^{38}(\mathrm{~d} / 3 \mathrm{kpc})^{2} \mathrm{erg} \mathrm{s}^{-1}$, to the end of the fireball stage ${ }^{3}$. An intermediate minimum of $R_{\mathrm{WD}}$ around Aug. 17.2 was probably caused by a transient decrease of the optical depth along the line of sight, resulting in a deeper view into the ejecta, down into a hotter optically thick shell, which radiates at unchanged $L_{\mathrm{WD}}$. On the other hand, a small transient increase of $R_{\mathrm{WD}}$ around Aug. 18.4 was accompanied by an increase in $L_{\mathrm{WD}}$ and a small decrease of $T_{\text {eff }}$ (see Fig. 3). This suggests that the outflowing material was inhomogeneous. Another striking result we obtained from our modelling of the SED is the very high luminosity of V339 Del, which is well above its Eddington limit for $M_{\mathrm{WD}} \sim 1.0 M_{\odot}$ (Chochol et al. 2014), during the fireball stage and beyond (Table 3 ). This result also contradicts the theoretical expectation that a super-Eddington phase only persists for a few hours at the very beginning of the nova eruption (e.g. Prialnik \& Kovetz 1995; Starrfield et al. 2008).

Nevertheless, the super-Eddington luminosity was previously observationally documented for some novae in the past. For example, Friedjung (1987) showed that the energy output of nova FH Ser was well above the Eddington limit for about two months after its optical maximum. Schwarz et al. (2001) demonstrated that the luminosity of the nova LMC 1991 was super-Eddington before its visual maximum and reached $L_{\mathrm{WD}} \sim$ $6 \times 10^{5} L_{\odot}$. Recently, Skopal (2014b) revealed this also for the recurrent nova RS Oph.

The super-Eddington state was theoretically investigated by Shaviv (1998), who considered a reduction of the effective opacity in the inhomogeneous atmosphere of novae, which increases the Eddington luminosity well above its classical value, calculated for the Thomson-scattering opacity. Lowering the effective opacity can result from the rise of a "porous layer" above the convective zone of the burning WD (see Fig. 1 of Shaviv \& Dotan 2010). Under these conditions novae evolve at a superEddington steady state, which can explain their very long decay times (Shaviv \& Dotan 2012). The long-term super-Eddington luminosity and the inhomogeneous mass ejecta, as we derived from our observations, seem to reflect Shaviv's theoretical predictions. However, further detailed multiwavelength analyses of other objects are required to allow for a more accurate theoretical modelling of the nova phenomenon.

Finally, a very large effective radius of the optically thick pseudophotosphere at the end of the fireball stage $\left(R_{\mathrm{WD}} \sim\right.$ $300 R_{\odot}$ ) suggests a high mass of the ejected material (Sect. 4.3).

\subsection{Biconical ionization structure of the fireball}

\subsubsection{Origin of the $\mathrm{H} \alpha$ line}

To understand the origin of the $\mathrm{H} \alpha$ line during the fireball stage of nova V339 Del, we need to identify (i) the ionizing source that is capable of giving rise to the observed $\mathrm{H} \alpha$ luminosity; and (ii) the material that is subject to ionization, the kinematics of which corresponds to the observed RVs.

\footnotetext{
A small decrease of $L_{\mathrm{WD}}$ at the end of the fireball stage was probably only apparent because of the disk-like shape of the pseudophotosphere (see Sect. 4.2.3), which does not allow estimating the total luminosity of the central source (see Sect. 5.3.6 of Skopal 2005).
} 


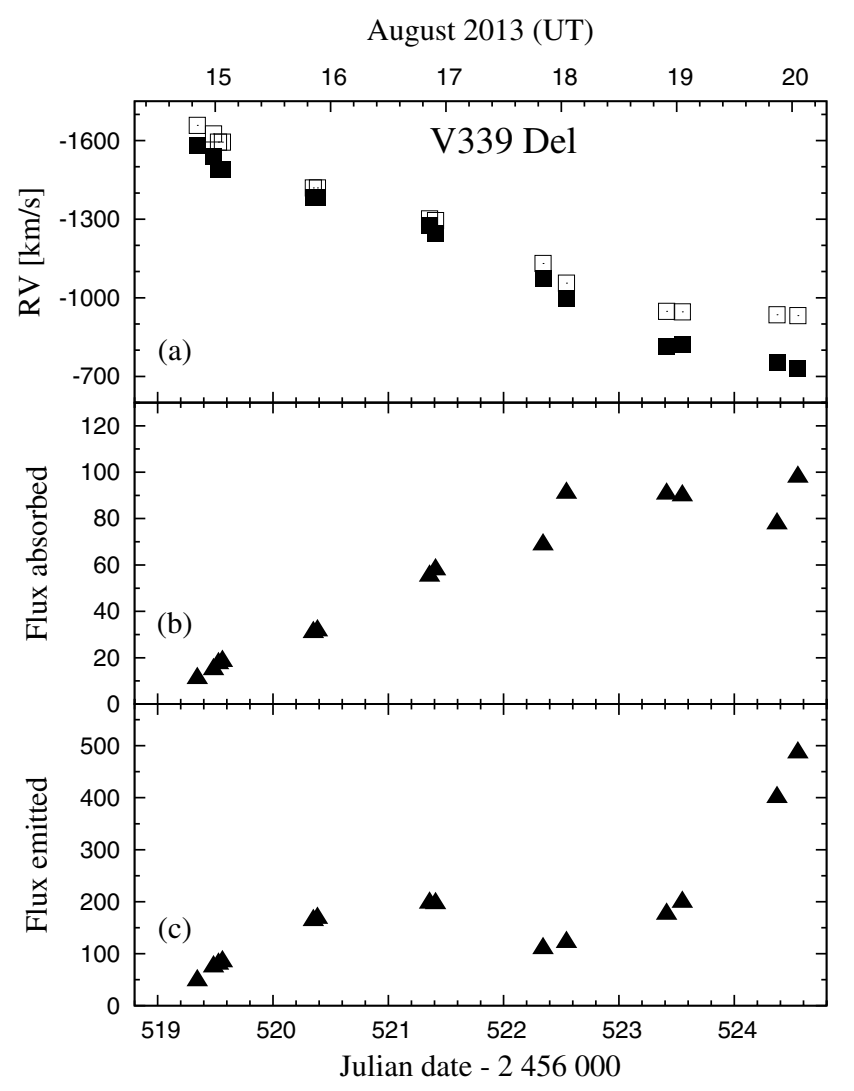

Fig. 7. Radial velocities and fluxes of the main components of the $\mathrm{H} \alpha$ profile as a function of time during the fireball stage (see Sect. 3.3). Fluxes are in $10^{-11} \mathrm{erg} \mathrm{cm}^{-2} \mathrm{~s}^{-1}$. a) Radial velocities of separated (filled squares) and observed (empty boxes) absorption components. b) Flux absorbed by the $\mathrm{H} \alpha$ line. c) Flux emitted by the separated components (from Aug. 18.91 on, it also includes the broad emission wing).

(i) Already the first spectra of V339 Del indicated the presence of rather strong emission lines of hydrogen. For example, the luminosity, $L_{\alpha}$, produced by the $\mathrm{H} \alpha$ line corrected for the absorption component was between $5.3 \times 10^{35}$ and $4.1 \times 10^{36}(\mathrm{~d} / 3 \mathrm{kpc})^{2} \mathrm{erg} \mathrm{s}^{-1}$, as measured on our first (Aug. 14.84) and last (Aug. 19.87) spectra during the fireball stage (Fig. 7). This luminosity was generated in a volume with the emission measure $E M_{\alpha}=L_{\alpha} / \varepsilon_{\alpha}=2.9 \times$ $10^{60}-2.2 \times 10^{61}(d / 3 \mathrm{kpc})^{2} \mathrm{~cm}^{-3}$ for the volume emission coefficient in $\mathrm{H} \alpha, \varepsilon_{\alpha}(20000 \mathrm{~K})=1.83 \times 10^{-25} \mathrm{erg} \mathrm{cm}^{3} \mathrm{~s}^{-1}$. This requires $L_{\mathrm{ph}}=\alpha_{\mathrm{B}} \times E M_{\alpha}=4.1 \times 10^{47}-3.1 \times 10^{48}$ ionizations/recombinations per second for the total hydrogen recombination coefficient $\alpha_{\mathrm{B}}(20000 \mathrm{~K})=1.43 \times 10^{-13} \mathrm{~cm}^{3} \mathrm{~s}^{-1}$ (Péquignot et al. 1991). However, the rate of hydrogenionizing photons, produced by the 6000-12000 warm pseudophotosphere of the nova, $L_{\mathrm{ph}}($ shell $)=2.4 \times 10^{41}-3.1 \times$ $10^{46}$ photons s $^{-1}$, is far below the rate of recombinations required to generate the $L_{\alpha}$ (i.e. $L_{\mathrm{ph}}($ shell $) \ll \alpha_{\mathrm{B}} \times E M_{\alpha}$ ). Thus the warm envelope cannot be ascribed to the ionizing source. This implies that the ionizing source is not seen directly by the observer, because the $E M_{\alpha}$ derived from the observed $L_{\alpha}$ is a few orders of magnitude higher than what can be produced by the warm pseudophotosphere. Such radiative components in the spectrum, which correspond to very different and mutually inconsistent temperature regimes, are often observed during outbursts of symbiotic stars. The corresponding spectrum is called a two-temperature-type spectrum (see Sect. 5.3.4 of Skopal 2005).

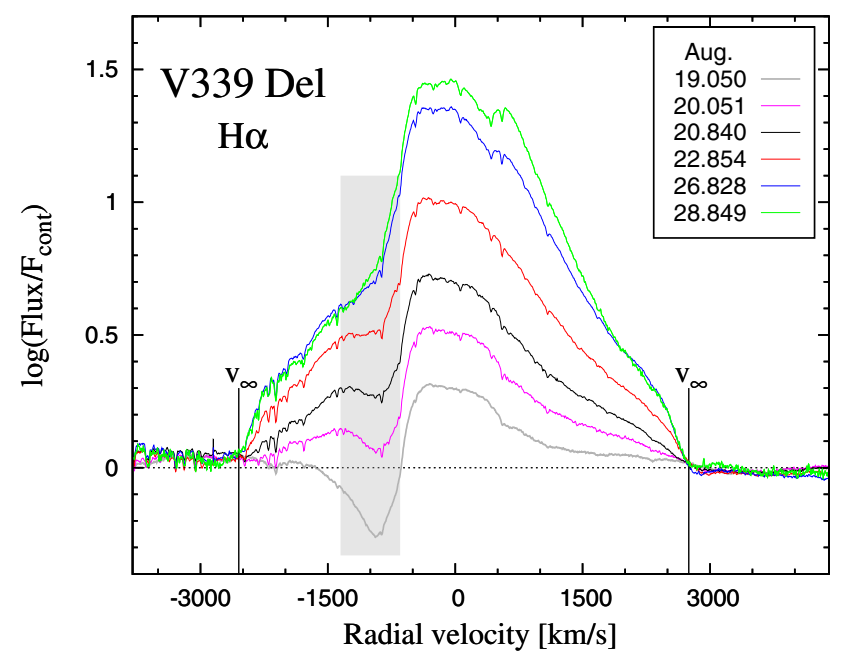

Fig. 8. Selected $\mathrm{H} \alpha$ profiles close to and after the end of the fireball stage. The grey belt illustrates the position of the absorption component. Terminal velocities of the emission wings are denoted by $v_{\infty}$.

(ii) An additional constraint for determining the ionizing source is provided by the broad $\mathrm{H} \alpha$ profile, the red wing of which extends to $\gtrsim 2000 \mathrm{~km} \mathrm{~s}^{-1}$ during the fireball stage. Approaching the end of this phase (after Aug. 18), the broad wing has strengthened and extended to a terminal velocity of $\sim 2700 \mathrm{~km} \mathrm{~s}^{-1}$ (Figs. 4 and 8). This implies that the mass outflow of the ionized hydrogen must have been collimated. Otherwise, the large optically thick envelope $\left(R_{\mathrm{WD}} \sim\right.$ $100-300 R_{\odot}$ ) would eclipse a significant fraction of the material flowing away from the observer, and would therefore preclude identifying it in the form of the extended red wing of the line.

Observational constraints included in points (i) and (ii) above imply that the mass outflow of nova V339 Del was not spherically symmetric during the fireball stage, meaning that the density was not homogeneously distributed in the envelope. Both the flux and the profile of the $\mathrm{H} \alpha$ line suggest that there is a low-density optically thin part, located in bipolar direction relative to the burning WD, probably along its rotation axis (i.e. perpendicular to its orbital plane). Its particles are ionized by the hot WD surface and driven out in the form of a fast wind. The remainder of the surrounding envelope is neutral. Its optically thick/thin interface represents the warm expanding pseudophotosphere (see top panel of Fig. 9). A similar ionization structure of the expanding envelope during the fireball phase was also indicated for RS Oph (see Fig. 4 of Skopal 2014b). In the case of V339 Del, the opening angle of the H II zone has to be narrower because the nebular continuum is significantly weaker than in the case of RS Oph.

\subsubsection{Variations of the $\mathrm{H} \alpha$ profile}

According to the biconical ionization structure of the envelope during the fireball stage, the flat to double-peaked emission component of the $\mathrm{H} \alpha$ line can be produced by the ionized material driven in bipolar directions from the burning WD surface. Each pole emission can be approximated by one component.

From Aug. 16 on, a strengthening of the absorption component, which reached its maximum around Aug. 18 (Sect. 3.3, Fig. 7), was probably caused by the inflation of the WD pseudophotosphere by a factor of $>2$. This was approximately consistent with the enlargement of the effective surface of the optically 


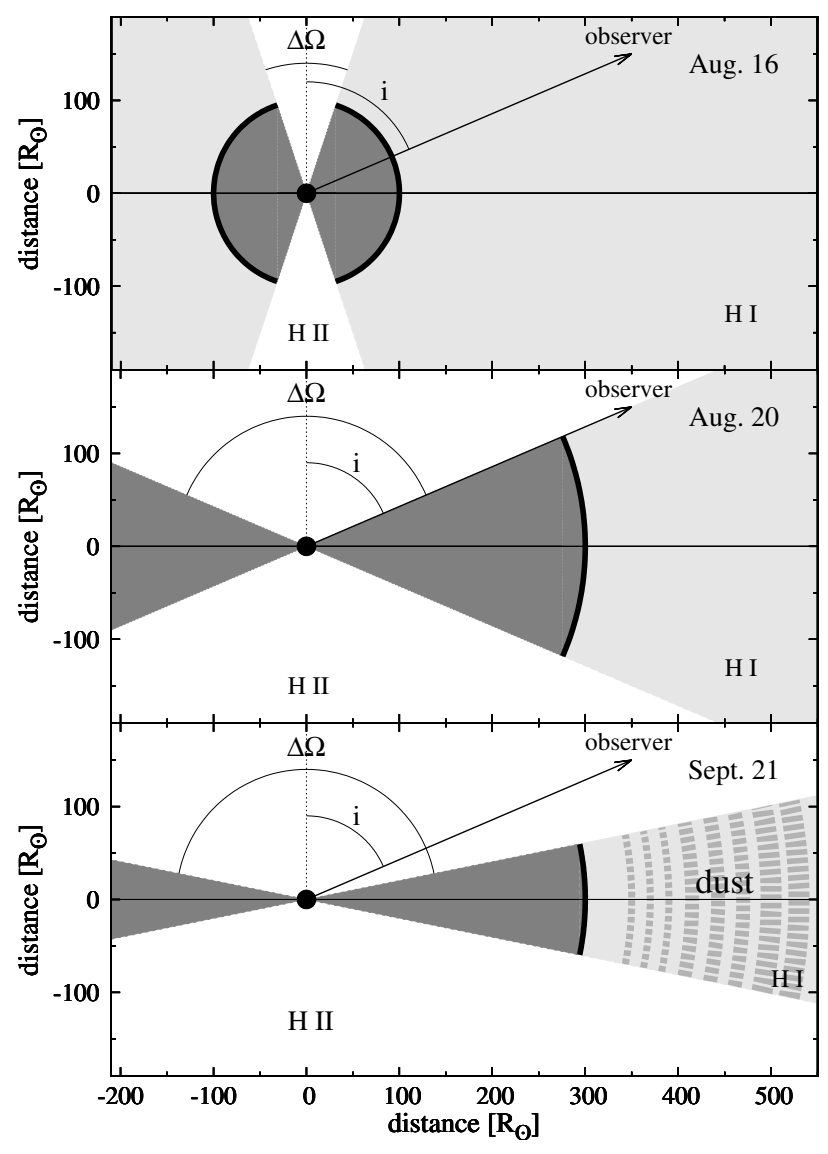

Fig. 9. Sketch of the ionization structure of nova V339 Del as seen on a cut perpendicular to the orbital plane that contains the burning WD (the black circle). The neutral H I zone is optically thick to the distance of the WD pseudophotosphere (heavy solid line). The ionized H II zone has the opening angle $\Delta \Omega$, and $i$ is the orbital inclination (its value is unknown). The top panel shows the structure around the beginning of the fireball stage, the middle one at its end, and the bottom panel shows the situation when the dust emission is present in the ejecta simultaneously with the H I atoms, hard radiation from the burning WD, and the large amount of the nebular emission (see Fig. 2, Sect. 4.2).

thick envelope (Table 3). A deceleration of the envelope expansion caused a gradual shift of the absorption component to longer wavelengths (see Figs. 4 and 7), which narrowed the $\mathrm{H} \alpha$ emission to a single peak. In addition, the significantly expanded pseudophotosphere eclipsed a larger part of the H II zone, which probably caused the transient decrease of its emission as observed around Aug. 18 (Fig. 7).

The increase of the $\mathrm{H} \alpha$ flux and the expansion of the emission wings of the line during the last day of the fireball stage (Figs. 4 and 8) was apparently caused by the biconical "opening" of the H II region (see below, Sect. 4.2.3).

\subsubsection{Disk-like shape of the envelope and its dilution}

During the last day of the fireball stage (Aug. 19), the emission produced by hydrogen lines was clearly increasing (see Fig. 7 for $\mathrm{H} \alpha$ ). For example, the total flux (i.e. corrected for the absorption component) in the $\mathrm{H} \alpha$ line increased from 1.8 to $3.9 \times 10^{-9} \mathrm{erg} \mathrm{cm}^{-2} \mathrm{~s}^{-1}$ from Aug. 18.91 to Aug. 19.87. In addition, the nebular continuum also became noticeable in our last spectrum taken during the fireball stage, on Aug. 19.89 (Fig. 1). In contrast, $T_{\text {eff }}$ and $L_{\mathrm{WD}}$ of the warm shell were decreasing and were much lower than what would be needed to produce the measured nebular emission, as described in Sect. 4.2.1. This implies that the unseen ionizing source was increasing. According to the biconical ionization structure of the expanding envelope (see Sect. 4.2.1), the gradual increase of the nebular contribution could be caused by a gradual opening of the H II zone, which would mean a narrowing of the H I zone. The optically thick envelope was therefore developing to a disk-like structure. Its outer flared rim represented the pseudophotosphere. From this point of view, the warm stellar component that dominates the spectrum implies that the ionizing source was hidden by the outer rim of the disk, that is, it was below the observer's horizon represented by the $\mathrm{HI} / \mathrm{H}$ II boundary. As soon as the opening angle of the $\mathrm{H}$ II zone becomes $>2 \times i$, the burning WD rises above the boundary and thus terminates the fireball stage (see Fig. 9). As a result, we can observe a hotter WD photosphere throughout an optically thinner H II zone, but a contribution from a remnant H I pseudophotosphere can also be expected (see the bottom panel of Fig. 9). This transition was demonstrated by the model SED of Aug. 21.7, which indicates a strong nebular component of radiation supplemented by a component from the warm residual pseudophotosphere and a hotter WD photosphere (Sect. 3.2.2, Fig. 2). In the $V$-light curve this was reflected by the first rapid fading from a flat maximum on Aug. 19 (see Fig. 2 of Munari et al. 2013a).

A significant decrease of $R_{\mathrm{WD}}$ from $\sim 300 R_{\odot}$ to $\leq 19 R_{\odot}$ in two days is consistent with a rapid decrease of the optical depth along the line of sight caused mainly by the geometrical effect of the rise of the hot WD above the $\mathrm{H} \mathrm{I} / \mathrm{H}$ II horizon (see Fig. 9). A similar effect of a sudden shift of the SED maximum to the ultraviolet was also indicated in the spectrum of nova RS Oph, which occurred during a much shorter time than the corresponding dynamical time-scale (see Sect. 4.2 of Skopal 2014b).

A gradual opening of the H II zone can be a result of a decreasing mass-loss rate from the WD (see Sect. 4.4). Recently, Cariková \& Skopal (2012) showed that the biconical ionization structure can be formed as a consequence of the enhanced massloss rate from the rotating WD during outbursts of symbiotic binaries. In their model, the opening of the $\mathrm{H}$ II zone is related to the mass loss rate from the WD as $\dot{M}_{\mathrm{WD}}^{-2}$ (see their Figs. 1 and 6).

Finally, there are two consequences of this type of the nova envelope dilution.

(i) The duration of the fireball stage can be a function of $i$. In general, a higher inclination implies a longer-lasting fireball stage, and vice versa. The transition of the maximum of the SED from the optical to the UV can occur during a short time of a few days.

(ii) The neutral material within the $\mathrm{H}$ I zone, which has the form of a disk encompassing the hot WD, can persist within the ejecta for a much longer time than the duration of the fireball phase until it becomes totally ionized. It is evident as an extra component in the model SEDs during a few days after the fireball stage (Fig. 2, Sect. 3.2.2). Further evident proof of the H I zone beyond the fireball stage is the Ramanscattered O VI $1032 \AA$ line in the optical spectrum for more than one month (Sect. 3.4).

\subsubsection{Probing the neutral disk by the Raman-scattering}

In the process of Raman-scattering, a photon excites an atom from its ground state to an intermediate state, which is immediately stabilized by a transition to a different true bound state. This process produces the Raman-scattered photon at a (very) 
different frequency and the corresponding rest line photon (see Fig. 1 of Nussbaumer et al. 1989).

The Raman-scattering of the OVI $1032 \AA$ line photons by neutral hydrogen atoms produces a broad emission band at $6825 \AA$. This Raman conversion was clearly indicated in the spectra of many symbiotic stars. Its efficiency depends on the scattering geometry; more accurately, on the fraction of the sky as seen from the O VI zone, which is covered by the neutral gas where Raman scattering can take place. A small cross-section of the $\lambda 1032 \rightarrow \lambda 6825$ Raman conversion, $\sigma_{\text {Ram }} \sim 4.4 \times 10^{-24} \mathrm{~cm}^{2}$ (Schmid 1989) requires a large amount of neutral material in the circumstellar environment where it originates. The column density of the $\mathrm{HI}$ atoms along the line of sight of the incident $\mathrm{O}$ VI photons must exceed a value of $N_{\mathrm{H}} \gtrsim 10^{23} \mathrm{~cm}^{-2}$ to produce an observable effect (i.e., the optical depth $N_{\mathrm{H}} \sigma_{\text {Ram }} \gtrsim 1$ ).

From the properties of the Raman-scattering process and its induced feature observed around $6830 \AA$ (see Fig. 5, Sect. 3.4) we can infer some characteristics of both the neutral $\mathrm{HI}$ and the ionized O VI zone.

(i) The Raman-scattered O VI $1032 \AA$ line in the spectrum of V339 Del confirms that there is a massive HI zone in a classical nova as well. This seems surprising, since there is no other source of neutral hydrogen, as there is in symbiotic binaries. However, according to the ionization structure of the envelope and its evolution during and after the fireball stage (Sects. 4.2.1 and 4.2.3), the Raman-scattering takes place in the neutral disk-like formation that encompasses the burning WD (see also Fig. 9).

(ii) The profile of the Raman line did not change markedly throughout its visibility. This implies that the geometry of the scattering $\mathrm{H}$ I region (i.e. the disk around the hot ionizing source) also persisted more or less unchanged.

(iii) A gradual decrease of the Raman flux since the end of August reflected a gradual decrease of the Ramanscattering efficiency due to a narrowing of the disk-like H I zone; as a consequence, the solid angle of sky area filled with neutral gas as seen from the $\mathrm{O}$ VI photon-emitting zone becomes smaller. This is probably a result of a decreasing mass-loss rate (see Sect. 4.4), which leads to a gradual ionization and hence flattening of the neutral disk (Cariková \& Skopal 2012), which in turn leads to an enlargement of the H II zone (see Sect. 4.3).

(iv) The structure of the Raman profile itself results from the relative motions between the main emitting and scattering regions. A redward shift of the Raman-scattered emission is consistent with its origin predominantly in the neutral disk that expands from the hot WD. A similar interpretation was suggested for the symbiotic prototype Z And during its 2006 post-outburst stage (see Fig. 5 of Skopal et al. 2009).

(v) Finally, the Raman-scattered O VI $1032 \AA$ line in the spectrum requires the existence of an ionizing source that is capable of producing the $\mathrm{O}^{+5}$ ions. According to Mürset \& Nussbaumer (1994), the ionization potential $\chi\left(\mathrm{O}^{+5}\right) \sim$ $114 \mathrm{eV}$ requires $T_{\mathrm{WD}} \sim 114000 \mathrm{~K}$. At this temperature, other permitted lines with lower ionization potential (e.g. He II lines) are also expected in the spectrum. However, no He II lines were identified at the same time as the Raman $6830 \AA$ emission. This is a puzzle. We can only speculate that the emission regions with highly ionized elements are very small (an example is given in Appendix B of Skopal et al. 2006), so that the surrounding dense layers can absorb photons of corresponding transitions. However, especially the O VI $1032 \AA$ photons can be effectively absorbed by Rayleigh and Raman scattering. The former is a multiplescattering resonance process (it generates a photon of the same frequency), which can be terminated by the latter one - the last transition in the Rayleigh-scattering chain, which produces the Raman $6825 \AA$ photon. As there is no other strong transition of any other element at/around this wavelength (e.g. Allen 1980), the Raman photon can easily escape from the region where it was created.

\subsection{Mass of the emitting material}

According to our modelling results for the SED, the nebular emission in the continuum arose at the end of the fireball stage, when the broad wings of the $\mathrm{H} \alpha$ line also expanded to $v_{\infty}= \pm 2650 \mathrm{~km} \mathrm{~s}^{-1}$ (Sect. 3.3, Fig. 8). Assuming that the hydrogen recombination lines are produced within the same emission region as the nebular continuum, its volume can be estimated as

$V_{\text {neb }}=\epsilon \frac{4}{3} \pi R_{\text {neb }}^{3}$,

where the filling factor $\epsilon<1$ reduces the spherical volume by taking into account the biconical structure of the H II zone due to the neutral disk that encompasses the WD (see Fig. 9). In addition, the disk blocks a fraction of the ionized region from its opposite side in the direction of the observer. The largest radius of the emitting volume is $R_{\text {neb }}=v_{\infty} \times t$, where $t$ is the time elapsed since the end of the fireball stage (Aug. 19.9, 2013). Then, according to the definition of the emission measure, the average particle density of the nebular-emitting region can be approximated as $\bar{n}=\left(E M / V_{\text {neb }}\right)^{1 / 2}$. With the aid of these simple relations, we can write the emitting mass of the nebula, $M_{\text {neb }}=\mu m_{\mathrm{H}} \bar{n} V_{\text {neb }}$, in the form

$M_{\mathrm{neb}}=\xi \times \epsilon^{1 / 2}\left(\frac{E M}{\left[\mathrm{~cm}^{-3}\right]}\right)^{1 / 2}\left(\frac{v_{\infty}}{\left[\mathrm{km} \mathrm{s}^{-1}\right]} \frac{t}{[\mathrm{~d}]}\right)^{3 / 2} M_{\odot}$,

where the factor $\xi=1.9 \times 10^{-42}$ for the mean molecular weight $\mu=1.4$ and the mass of the hydrogen atom $m_{\mathrm{H}}=1.67 \times 10^{-24} \mathrm{~g}$.

On Aug. $22(t \sim 2$ days $)$, the model $E M=2 \times$ $10^{62}(\mathrm{~d} / 3 \mathrm{kpc})^{2} \mathrm{~cm}^{-3}$ corresponded to a maximum $(\epsilon=1)$ mass of the emitting material, $M_{\text {neb }} \sim 1 \times 10^{-5} M_{\odot}$. The subsequent quantities of $M_{\text {neb }}$ derived for Aug. 28 ( $t \sim 9$ days), Sept. 13 $(t \sim 25$ days $)$, and Sept. 20 ( $t \sim 31.5$ days $)$ were increasing to $9.3 \times 10^{-5}, 3.8 \times 10^{-4}$, and $4.6 \times 10^{-4} M_{\odot}$, respectively (for $E M$ in Table 3). Such a large increase of $M_{\text {neb }}$ could not only be caused by a high mass-loss rate from the burning WD (see below), but mainly by a gradual dilution (i.e. ionization) of the neutral disk-like material, as indicated by the evolution of the Raman 6825 A O VI line (Fig. 5).

Considering only the biconical shape of the emitting region, the filling factor $\epsilon=2 \Delta \Omega / 4 \pi$, where $\Delta \Omega$ is the opening angle of the H II region in sr. For example, $\Delta \Omega=\pi$ gives $\epsilon=0.5$, which lowers $M_{\text {neb }}$ by a factor of 0.7 . The very high EM suggests a large $\Delta \Omega$ with $\epsilon$ close to 1 , and thus no significant reduction of $M_{\text {neb }}$ due to the filling factor in Eq. (4) can be expected.

\subsection{Mass-loss rate of the ionized material}

After the fireball stage, the nebular component of radiation dominated the optical to near-IR spectrum of V339 Del (Fig. 2). It represents a fraction of the WD's radiation re-processed by ionization processes followed by recombinations and free-free transitions. Thus, knowing the physical process and its result, the 
EM from the model SED allows us to determine the mass-loss rate of the ionized material, $\dot{M}_{\mathrm{WD}}$.

In our simplified approach the material flows out at a constant velocity $v_{\infty}$, and its density distribution $n(r)$ at a radial distance $r$ from its source satisfies the mass continuity equation as

$\dot{M}_{\mathrm{WD}}=2 \Delta \Omega r^{2} \mu m_{\mathrm{H}} n(r) v_{\infty}$,

where $\Delta \Omega<2 \pi$ is the opening angle of the HII region. It is further assumed that all particles become ionized on their path from the place of origin, $R_{\mathrm{WD}}$, to the end of the nebula, $R_{\mathrm{neb}}$. Then, in the case of a pure hydrogen gas, we can approximate the equilibrium condition between the flux of ionizing photons, $L_{\mathrm{ph}}$, and the rate of recombinations in the nebula as

$L_{\mathrm{ph}}(\mathrm{H})=\alpha_{\mathrm{B}}\left(\mathrm{H}, T_{\mathrm{e}}\right) \int_{\mathrm{HII}} n_{\mathrm{p}}(r) n_{\mathrm{e}}(r) \mathrm{d} V$

where $\alpha_{\mathrm{B}}\left(\mathrm{H}, T_{\mathrm{e}}\right)\left(\mathrm{cm}^{3} \mathrm{~s}^{-1}\right)$ is the recombination coefficient to all but the ground state of hydrogen (see also Eq. (4) of Nussbaumer \& Vogel 1987). This equation is valid for a hydrogen plasma heated by photoionizations and characterized by a constant $T_{\mathrm{e}}$. According to the geometry of the H II region, $\mathrm{d} V=2 \Delta \Omega r^{2} \mathrm{~d} r$. For a complete ionization $\left(n_{\mathrm{e}}(r)=n_{\mathrm{p}}(r)=n(r)\right)$, the equilibrium Eq. (6) can be integrated from $R_{\mathrm{WD}}$ to $R_{\mathrm{neb}}$, and using $n(r)$ given by Eq. (5), $\dot{M}_{\mathrm{WD}}$ can be expressed as

$\dot{M}_{\mathrm{WD}}=\left[2 \Delta \Omega\left(\mu m_{\mathrm{H}} v_{\infty}\right)^{2} \frac{L_{\mathrm{ph}}(\mathrm{H})}{\alpha_{\mathrm{B}}\left(\mathrm{H}, T_{\mathrm{e}}\right)}\left(\frac{1}{R_{\mathrm{WD}}}-\frac{1}{R_{\mathrm{neb}}}\right)^{-1}\right]^{1 / 2} \mathrm{gs}^{-1}$,

where the ratio $L_{\mathrm{ph}}(\mathrm{H}) / \alpha_{\mathrm{B}}\left(\mathrm{H}, T_{\mathrm{e}}\right)=E M$.

On Aug. 22, the parameters of the model SED, $R_{\mathrm{WD}}=19 R_{\odot}$, $R_{\text {neb }}=4.6 \times 10^{13} \mathrm{~cm}$ and $E M=2 \times 10^{62}(d / 3 \mathrm{kpc})^{2} \mathrm{~cm}^{-3}$ yield $\dot{M}_{\mathrm{WD}}=5.7 \times 10^{-4} M_{\odot} \mathrm{yr}^{-1}$. On Aug. 28, Sept. 13, and 20, $\dot{M}_{\mathrm{WD}}$ was decreasing to $4.5,1.4$, and $0.71 \times 10^{-4} M_{\odot} \mathrm{yr}^{-1}$, respectively (parameters in Table 3 ). In all cases we adopted $2 \Delta \Omega=4 \pi$ and $v_{\infty}=2650 \mathrm{~km} \mathrm{~s}^{-1}$ (Sect. 3.3), which correspond to maximum values of $\dot{M}_{\mathrm{WD}}$.

\section{Summary}

We modelled the optical/near-IR SED of the classical nova V339 Del from its discovery on Aug. 14, 2013 until the end of the first plateau phase in the light curve (around day 40). We determined the fundamental parameters $L_{\mathrm{WD}}, R_{\mathrm{WD}}$, and $T_{\mathrm{eff}}$ of the burning WD pseudopotosphere (Fig. 3). Monitoring the evolution of the $\mathrm{H} \alpha$ line profile and the transient emergence of the Raman-scattered $1032 \AA$ line in the optical spectrum allowed us to determine the ionization structure of the nova during this early period of its evolution (Fig. 9). The main results of our analysis may be summarized as follows.

1. During the fireball stage (Aug. 14.84-19.89, 2013; see Sect. 3.1), $T_{\text {eff }}$ was in the range of $6000-12000 \mathrm{~K}, R_{\mathrm{WD}}$ was expanding in a non-uniform way from $\sim 66$ to $\sim 300$ $(d / 3 \mathrm{kpc}) R_{\odot}$ and $L_{\mathrm{WD}}$ was super-Eddington, but not constant. Its maximum occurred around 1.5 day after the nova discovery, at the maximum of $T_{\text {eff }}$ (Aug. 16.0), when $L_{\mathrm{WD}}$ rapidly increased by a factor of $\sim 6$ with respect to its initial value of $\sim 1.5 \times 10^{38}(d / 3 \mathrm{kpc})^{2} \mathrm{erg} \mathrm{s}^{-1}$ (Sect. 3.2.1, Fig. 3). Thus, V339 Del did not obey the theoretical prediction that a nova evolves at a constant bolometric luminosity in the early stages of outburst (Sect. 4.1).
2. After the fireball stage ( $\gtrsim$ Aug. 20), the optical/near-IR SED changed significantly (Figs. 1 and 2). The continuum was dominated by the nebular radiation component with a large $E M=1.0-2.0 \times 10^{62}(d / 3 \mathrm{kpc})^{2} \mathrm{~cm}^{-3}$. As a result, only limiting values of the $L, R, T$ parameters could be determined from the measured $E M$ and the profile of the SED (Sect. 3.2.2). The lower limit of $L_{\mathrm{WD}}$ was still superEddington, being around $1 \times 10^{39}(d / 3 \mathrm{kpc})^{2} \mathrm{erg} \mathrm{s}^{-1}$, which is higher than the value we derived during the fireball stage.

3. The profile of the $\mathrm{H} \alpha$ line and its relative high flux even during the fireball phase imply that there is a low-density optically thin part of the envelope, with a bipolar shape relative to the burning WD. This part of the envelope is ionized, while the remainder is neutral. The optically thick/thin interface represents the warm expanding pseudophotosphere. A gradual increase of the nebular contribution during the last day of the fireball stage (Aug. 18.9-19.9) could be caused by a gradual opening and enlargement of the H II zone, causing a narrowing of the HI zone. The optically thick pseudophotosphere was therefore transformed into a disk-like shape (Sect. 4.2, Fig. 9).

4. According to the biconical ionization structure of the nova ejecta, the rapid decrease of $R_{\mathrm{WD}}$ from $\sim 300$ to $\sim 19 R_{\odot}$ and the dramatic change in the SED during a few days after the fireball stage was caused by the rising of the hot WD above the $\mathrm{HI} / \mathrm{H}$ II horizon with respect to the line of sight (Fig. 2, Sect. 4.2.3).

5. A disk-like HI region was indicated within the ejecta for a period of $\gtrsim 1$ month after the fireball stage, until its total ionization, around day 40. The most conclusive proof of the presence of the $\mathrm{HI}$ zone around the burning WD is the presence of the Raman-scattered O VI $6830 \AA$ emission, which requires a column density of the $\mathrm{HI}$ atoms of $N_{\mathrm{H}} \gtrsim 10^{23} \mathrm{~cm}^{-2}$ (Sect. 4.2.4, Fig. 5).

6. On Sept. 20, our model SED indicated a dust emission in the near-IR. The simultaneous presence of the hard radiation from the burning WD constrains the dust to be located in a ring beyond the H I zone, where it is shielded from the hightemperature radiation (bottom panel of Fig. 9).

7. The emitting mass of the $\mathrm{H}$ II zone was gradually growing from $\sim 1 \times 10^{-5}$ (Aug. 22) to $\sim 4.6 \times 10^{-4} M_{\odot}$ (Sept. 20) mainly due to a gradual ionization of the H I zone (Sect. 4.3).

8. The mass-loss rate from the nova was decreasing from $\sim 5.7 \times$ $10^{-4}$ on Aug. 22 to $\sim 7.1 \times 10^{-5} M_{\odot} \mathrm{yr}^{-1}$ on Sept. 20 (Sect. 4.4).

As in the case of the extraordinary classical nova LMC 1991 (Schwarz et al. 2001) and/or the symbiotic recurrent nova RS Oph (Skopal 2014b), the special physical conditions derived from observations of the classical nova V339 Del represent new challenges for the theoretical modelling of the nova phenomenon.

Acknowledgements. We thank the anonymous referee for constructive comments. The spectra presented in this paper were in part obtained within the Astronomical Ring for Access to Spectroscopy (ARAS), an initiative promoting cooperation between professional and amateur astronomers in the field of spectroscopy. The authors thank ARAS observers for their contributions made within the ARAS programme coordinated by Francois Teyssier. We also acknowledge the variable-star observations from the AAVSO International Database contributed by observers worldwide and used in this research. This research has been in part supported by the project No. SLA/103115 of the Alexander von Humboldt foundation and by a grant of the Slovak Academy of Sciences VEGA No. 2/0002/13. 


\section{References}

Allen, D. A. 1980, MNRAS, 190, 75

Burlak, M. A., Shenavrin, V. I., Tatarnikov, A. M., \& Tatarnikova, A. A. 2013, ATel, 5294

Cardelli, J. A., Clayton, G. C., \& Mathis, J. S. 1989, ApJ, 345, 245

Cariková, Z., \& Skopal, A. 2012, A\&A, 548, A21

Cass, A. C., Carlon, R. L., Corgan, D. T., et al. 2013a, ATel, 5317

Cass, A. C., Carlon, R. L., Corgan, D. T., et al. 2013b, ATel, 5340

Cass, A. C., Carlon, R. L., Corgan, D. T., et al. 2013c, ATel, 5434

Chochol, D., Shugarov, S., Pribulla, T., \& Volkov, I. 2014, Contrib. Astron. Obs. Skalnaté Pleso, 43, 1

Cox, A. N. 2000, Allen's Astrophysical Quantities, 4th edition (New York: AIP Press, Springer)

Darnley, M. J., \& Bode, M. F. 2013, ATel, 5300

Darnley, M. J., Bode, M. F., Smith, R. J., \& Evans, A. 2013, ATel, 5279

Friedjung, M. 1987, A\&A, 179, 164

Gehrz, R. D., Dykhoff, D. A., \& Shenoy, D. P. 2013, ATel, 5299

Henden, A. A., \& Kaitchuck, R. H. 1982, Astronomical Photometry, (New York:

Van Nostrand Reinhold Company), 50

Munari, U., Sordo, R., Castelli, F., \& Zwitter, T. 2005, A\&A, 442, 1127

Munari, U., Henden, A., Dallaporta, S., \& Cherini, G. 2013a, IBVS No. 6080

Munari, U., Valisa, P., Milani, A., \& Cetrulo, G. 2013b, ATel, 5297

Mürset, U., \& Nussbaumer, H. 1994, A\&A, 282, 586

Nussbaumer, H., \& Vogel., M. 1987, A\&A, 182, 51

Nussbaumer, H., Schmid, H. M., Vogel, M. 1989, A\&A, 211, L27

Page, K. L., Osborne, J. P., Kuin, N. P. M., et al. 2013, ATel, 5470

Péquignot, D., Petitjean, P., \& Boisson, C. 1991, A\&A, 251, 680

Prialnik, D., \& Kovetz, A. 1995, ApJ, 445, 789
Schmid, H. M. 1989, A\&A, 211, L31

Schwarz, G. J., Shore, S. N., Starrfield, S., et al. 2001, MNRAS, 320, 103

Shaviv, N. J. 1998, ApJ, 494, L193

Shaviv, N. J., \& Dotan, C. 2010, Mem. Soc. Astron. It., 81, 350

Shaviv, N. J., \& Dotan, C. 2012, Mem. Soc. Astron. It., 83, 792

Shore, S. N., Sonneborn, G., Starrfield, S., Gonzalez-Riestra, R., \& Polidan, R. S. 1994, ApJ, 421, 344

Shenavrin V. I., Taranova O. G., \& Tatarnikov A. M. 2013, ATel, 5431

Shore, S. 2008, in Classical Novae, eds. M. F. Bode, \& A. Evans (Cambridge: CUP), 194

Shore, S., Škoda, P., \& Rutsch, P. 2013a, ATel, 5282

Shore, S., Škoda, P., Korčáková, D., et al. 2013b, ATel, 5312

Shore, S., Alton, K., Antao, D., et al. 2013c, ATel. No. 5378

Skopal, A. 2005, A\&A, 440, 995

Skopal, A. 2007, New Astron., 12, 597

Skopal, A. 2014a, New Astron., submitted [arXiv: 1401.1848]

Skopal, A. 2014b, New Astron., in press [arXiv: 1402 .6126]

Skopal, A., Vittone, A. A., Errico, L., et al. 2006, A\&A, 453, 279

Skopal, A., Pribulla, T., Budaj J., et al. 2009, ApJ, 690, 1222

Skopal, A., Tarasova, T. N., Cariková, Z., et al. 2011, A\&A, 536 A27

Skopal, A., Wolf, M., Šlechta. M., et al. 2014, ATel, 6132

Starrfield, S., Iliadis, Ch., Hix, W. R. 2008, in Classical Novae, eds. M. F. Bode, \& A. Evans (Cambridge: CUP), 77

Tarasova, T. N. 2013, ATel, 5291

Tarasova, T. N., \& Shakhovskoi, D. N. 2013, ATel, 5370

Tomov, T., Ilkiewicz, K., Swierczynski, E., Belcheva, M., \& Dimitrov, D. 2013, ATel, 5288

Warner, B. 2008, in Classical Novae, eds. M. F. Bode, \& A. Evans (Cambridge: CUP), 16 